\title{
Jung, feindselig, rechts!? Menschenfeindliche, rechtspopulistische und -extreme Orientierungen im intergenerativen Vergleich
}

\author{
Andreas Zick • Wilhelm Berghan • Nico Mokros
}

Eingegangen: 15. Januar 2020 / Überarbeitet: 20. Mai 2020 / Angenommen: 6. Oktober 2020 / Online publiziert: 26. Oktober 2020

(C) Der/die Autor(en) 2020

Zusammenfassung Im Hinblick auf den Umgang mit Rechtsextremismus und anderen antidemokratischen Orientierungen ist $\mathrm{zu}$ beobachten, dass diese häufig als ,Jugendproblem' wahrgenommen und verhandelt werden. Insbesondere, wenn rechtsextreme Straftaten von Heranwachsenden verübt werden. Große Teile pädagogischer Praxis der Demokratieförderung und Rechtsextremismusprävention richten sich an junge Menschen bzw. junge Erwachsene und auch die erziehungswissenschaftliche Forschung behandelt traditionell Rechtsextremismus in weiten Teilen im Rahmen der Jugendforschung. Selten wird die Frage gestellt, ob es tatsächlich eine empirisch nachweisbare Konzentration antidemokratischer Orientierungen in dieser Altersgruppe gibt. Dies zu wissen, wäre für Grundfragen nach den Bedingungen und Möglichkeiten politischer Sozialisation und Bildung relevant und ebenso wäre es fatal, wenn angesichts der Häufung bestimmter Phänomene in einer demografischen Gruppe ein Stereotyp in die Forschung einzieht. Der Beitrag hat daher ein recht einfaches Anliegen. Er stellt empirische Beobachtungen aus einer aktuellen bevölkerungsrepräsentativen Umfrage bereit und prüft statistisch, ob es zwischen menschenfeindlichen, rechtspopulistischen und rechtsextremen Einstellungen, die Orientierungen bilden, Unterscheide zwischen Altersgruppen gibt. Die Analyse zeigt, dass 18- bis 30-Jährige im Vergleich zu älteren Generationen zwar einzelnen rechtsextremen Einstellungsdimensionen auffallend zustimmen, nicht jedoch in der ganzen Breite menschenfeindlicher Einstellungen. In Bezug auf diese zeigen Ältere

Prof. Dr. A. Zick $(\bowtie) \cdot$ W. Berghan · N. Mokros

Institut für interdisziplinäre Konflikt- und Gewaltforschung sowie Fakultät für Erziehungswissenschaft, Universität Bielefeld, Universitätsstraße 25, 33615 Bielefeld, Deutschland E-Mail: zick@uni-bielefeld.de

W. Berghan

E-Mail: wberghan@uni-bielefeld.de

N. Mokros

E-Mail: n.mokros@uni-bielefeld.de 
höhere Zustimmung. Die Auswertungen legen nahe, die Fokussierung auf jüngere Altersgruppen und ihre politische Sozialisation viel enger und genauer in den Kontext der empirisch beobachtbaren gesellschaftlichen Stimmungen zu setzen.

Schlüsselwörter Gruppenbezogene Menschenfeindlichkeit · politische Sozialisation · Rechtsextremismus · Rechtspopulismus · Vorurteile

\title{
Young, hostile and right-wing? Hostile, right-wing, populist and extremist orientations in an intergenerational comparison
}

\begin{abstract}
Right-wing extremism and other anti-democratic orientations are often perceived and negotiated as a 'youth problems', especially when right-wing extremist crimes are committed by adolescents. Pedagogical practice for promoting democracy and preventing right-wing extremism are largely aimed at young people and young adults and educational research traditionally deals with right-wing extremism to a large extent within the framework of youth research. The question is rarely asked whether there actually is an empirically verifiable concentration of anti-democratic orientations in this age group. Knowing this is relevant for basic questions about the conditions and possibilities of political socialisation and education, it would also be important to ascertain if, given the accumulation of certain extremist phenomena in a demographic group, a stereotype has entered research. This article therefore has a rather simple purpose. It provides empirical observations from a recent population-representative survey and statistically examines whether there are differences between age groups between anti-human, right-wing populist and right-wing extremist attitudes that form orientations. The analysis shows that $18-$ to 30-year-olds, in comparison to older generations, agree strikingly with individual extreme right-wing attitudes, but not across the entire spectrum of misanthropic attitudes. Regarding these attitudes, older people show greater agreement. The evaluations suggest that, in the context of empirically observable social moods, the focus on younger age groups and their political socialisation should be much more closely and precisely placed.
\end{abstract}

Keywords Group-focused enmity $\cdot$ Political socialisation $\cdot$ Prejudice $\cdot$ Right-wing extremism $\cdot$ Right-wing populism

\section{Menschenfeindlichkeit und Rechtsextremismus - ein Jugendproblem?}

Wenn Gewalt und schwere Hasstaten gegen Mitglieder bestimmter Gruppen in der Gesellschaft als ,jugendlicher Rechtsextremismus“ sichtbar wird und dieser mediale, politische oder auch wissenschaftliche Aufmerksamkeit erfährt, dann zumeist und zunächst mit dem Fokus auf das Lebensalter und die Lebenslage der Täter_innen als Erklärungsfaktor. Für die Auseinandersetzung mit Menschenfeindlichkeit und Rechtsextremismus als ,Jugendproblem“ schien auch seit den rassistischen Ausschreitungen in den 90er-Jahren die Evidenz zu sprechen, dass es nach dem Mauerfall eine wachsende jugendkulturelle Szene um neonazistische Gruppierungen gab, 
die für die Angriffsserie auf Ausländer und Asylsuchende verantwortlich waren. Die Beschäftigung mit Rechtsextremismus war seitdem in weiten Teilen Jugendforschung (Rieker 2006).

Dabei wurde die Debatte von einem selektiven Verständnis geprägt, das sich auf jugendliche Täterbeschreibungen ausrichtete und vor allem junge Männer in Ostdeutschland meinte (Groffmann 2001; Jäger 1997; Lynen von Berg 2000). Insbesondere am Beispiel des sogenannten Nationalsozialistischen Untergrunds lässt sich nachzeichnen, dass deren Mitglieder sich im Jugend- bzw. jungen Erwachsenenalter radikalisierten (Würstl 2016). Und auch heute inszenieren sich junge Rechtsextreme stärker in der Öffentlichkeit als andere. Angesicht eines anhaltenden antidemokratischen Potenzials in der Gesellschaft wurde ein solch generations- bzw. jugendbezogenes Verständnis der betreffenden Phänomenbereiche teilweise neu begründet. Wie die Forschung zum Rechtsextremismus zunehmend junge Menschen in den Fokus nahm, zielte auch die mehr oder minder gut begründete Pädagogik gegen Rechts auf junge Menschen ab. Die aufgelegten Bundesprogramme zur Extremismusprävention und Demokratieförderung wurden verstärkt und im Feld pädagogischer Arbeit überwiegend an Kinder, Jugendliche, junge Erwachsene und deren Bezugspersonen gerichtet (vgl. BMFSFJ 2016). ${ }^{1}$

Obgleich dies noch zu erforschen wäre, entstehen dabei Verzerrungen und Wechselwirkungen zwischen der staatlichen Förderung einzelner Maßnahmen und Programme, der medialen Berichterstattung über Menschenfeindlichkeit und Rechtextremismus und eben auch einer erziehungswissenschaftlichen Konzentration auf das Jugendalter als Erklärungsfaktor. Letzteres spiegelt sich grundlegend in der Entwicklung von Theorie- und Forschungsansätzen wider, die sich auf sozio-ökonomische Prozesse der Modernisierung, Individualisierung und Desintegration stützten (vgl. Überblick von Frindte et al. 2016). Zementiert wurde damit der Eindruck, dass Jugendforschung vor allem Bereiche betritt, ,in denen Gesellschaft über Jugend beunruhigt ist, wo die Genese einer demokratisch gesonnenen Persönlichkeit auszubleiben droht" (Reinders 2001, S. 9). Mudde (2014) betont sogar, dass es mehr Studien zum Thema Jugend und Rechtsextremismus in Deutschland gibt als in der restlichen Welt zusammen.

Es ist nachvollziehbar und notwendig, dass die Entwicklung ,jugendlichen Rechtsextremismus ' in seinen verschiedenen Ausprägungsformen von Interesse für die Öffentlichkeit und Politik eines demokratisch verfassten Staats ist, der ausdrücklich einen Bildungs- und Erziehungsauftrag im Sinne demokratischer Grundsätze formuliert (z. B. KMK 2018). Allerdings gerät dabei der gesellschaftliche Kontext, in dem sich die politische Sozialisation auf der Grundlage geteilter Normen und Werte vollzieht, leicht aus dem Blick, wie schon Klönne (1994) auf die Frage verwies, ,wie denn jugendspezifische rechtsextreme Risiken und in der ,erwachsenen “ Gesamtgesellschaft etablierte Weltbild und Verhaltensweisen ineinandergebunden sind“ (S. 141). Auch die Analyse von Rieker (2006) verweist darauf.

Mit der im Jahr 2014 wieder aufgekeimten ,(Anti-)Asyldebatte', deren tiefgreifenden Polarisierung in der Gesellschaft und Resonanz für antidemokratische, wie

\footnotetext{
${ }^{1}$ Im Jahr 2020 wird das Projekt MAPEX ein Online-Mapping aller geförderten Deradikalisierungs- und Extremismusprojekte bereitstellen, wo dies empirisch leicht zu überprüfen ist (www.mapex-projekt.de).
} 
menschenfeindliche und verschwörungsideologische Agitation ging ein enormer Anstieg des Personenpotentials sowie von Hass- und Gewalttaten im Bereich des Rechtsextremismus einher, bei gleichzeitig schwindender Abgrenzung davon in anderen Teilen der Bevölkerung (BMI 2016). Trotz oder wegen der gesellschaftlichen Verbreitung entsprechender Einstellungen, schienen aber vor allem bestimmte Altersgruppen, vermutlich sogar im Namen einer schweigenden Mehrheit, zur Tat zu schreiten. Länderspezifische Hellfeldstatistiken zur politisch-rechts-motivierten Kriminalität ${ }^{2}$ zeigen unter anderem, dass 2018 am häufigsten gegen männliche Personen über 21 bzw. 30 Jahren Tatverdacht bestand (Landeskriminalamt SachsenAnhalt 2019; Ministerium des Innern des Landes Nordrhein-Westfalen 2019), womit eher junge Erwachsene in den Fokus der Ermittlungen rückten.

Ähnliche Muster zeigen sich mit Blick auf das Wahlverhalten. Beispielsweise weisen Analysen der Landtagswahlen 2019 in Brandenburg, Sachsen und Thüringen auf entscheidende Verschiebungen zwischen den Altersgruppen hin. Entgegen des Bildes ,alter weißer Männer', die ihre Wut über einen vermeintlichen Bedeutungsund Privilegienverlust in der Unterstützung antifeministischer und rassistischer Kräfte ausdrücken (vgl. Kimmel 2016), waren es die über 60-Jährigen, die beispielsweise zu einem deutlich geringeren Anteil die AfD gewählt haben als die 30- bis 59-Jährigen. Gleichwohl erhielt die AfD mit durchgängigem Abstand die meisten Stimmen von männlichen Wählern (infratest dimap 2019a, b, c; Forschungsgruppe Wahlen 2019a, b, c).

Zur Untersuchung von antidemokratischen Einstellungen unter jungen Menschen rekurriert in Teilen auch die Shell-Jugendstudie auf einzelne Fragen jener Erhebung, die wir später zugrunde legen. Die darin befragten 12- bis 25-Jährigen äußern zuletzt ein nach 2015 anhaltendes Problembewusstsein für ,Ausländerfeindlichkeit", wenngleich rund $30 \%$ angeben, dass ihnen, die Zuwanderung nach Deutschland ' Angst macht. Ähnlich ambivalent wird die Aufnahme von Flüchtlingen bewertet und auch die Unterstellung eines Meinungsdiktats findet in der Aussage „In Deutschland darf man nichts Schlechtes über Ausländer sagen, ohne gleich als Rassist beschimpft zu werden" breite Zustimmung (Schneekloth und Albert 2019).

Konkret nach rechtsextremen Einstellungen gefragt, berichten Krieg und Kliem (2019) im Zeitvergleich zwischen 2013, 2015 und 2017 von Schüler_innen der 9. Jahrgangsstufe in Niedersachsen kaum Veränderungen für die Subdimension der Ausländerfeindlichkeit; mit den zwar höchsten Zustimmungswerten jedoch einen leichten Rückgang bei der erhobenen Form von Muslimfeindlichkeit; sowie eine deutlich geringe Zustimmung bei Antisemitismus und auch beim Chauvinismus. Eine rechtsautoritäre Diktatur befürworten auch die Wenigsten ohne signifikante Veränderungen über die Erhebungsjahre. Für 2015 und 2017 sei insgesamt eine höhere Prävalenz von Rechtsextremismus unter Jungen niedrigerer Schulformen als unter Mädchen höherer Schulformen festzustellen. Für weitere Vergleiche zu rechtsextremen Einstellungen sei auch auf die Analysen von Stöss (2007) sowie

\footnotetext{
${ }^{2}$ Die Aussagekraft von behördlicher Kriminalstatistik ist eingeschränkt, da sie ausschließlich das Anzeigeverhalten wiedergibt und der grundlegenden Erfassungsschwierigkeit unterliegt, dass z. B. rechtsextreme Motivlagen nachweisbar tatauslösend und tatbestimmend gewesen sind. Es bleibt davon auszugehen, dass Fallzahlen aus dem Dunkelfeld deutlich höher ausfallen (Baier et al. 2009).
} 
Stöss und Niedermayer (2008) verwiesen, die eher unter älteren Generationen höhere Zustimmungswerte verzeichneten.

Ebenso konnte mit Blick auf Vorurteilsmuster bzw. gruppenbezogene Menschenfeindlichkeit die Annahme altersspezifischer und veränderter Sozialisationsbedingungen bestätigt werden, dass im Vergleich zu jüngeren Befragten zwischen 16 und 25 Jahren fast durchgängig die Älteren über 26 Jahren den einzelnen Abwertungselementen signifikant häufiger zustimmten. Eine Ausnahme bildet die Abwertung von Obdachlosen. Keinen Unterschied zwischen den beiden Altersgruppen gab es bei der Abwertung von Menschen mit Behinderung (Endrikat 2006).

Auffällige (Geschlechts- und) Altersunterschiede herauszustellen scheint dem Forschungsstand zufolge durchaus angebracht, aber eine Vereinfachung und Verengung des Diskurses läuft Gefahr, die politische Sozialisation falsch zu verstehen und das antidemokratische Potenzial in der Breite der Bevölkerung zu unterschätzen, was immer wieder zur Selbstentlastung beiträgt. ${ }^{3}$ So vielschichtig und zeitgeschichtlich der Ausdruck von sozialen und politischen Ideologien der Ungleichwertigkeit ist, so unterschiedlich sind auch das Ausmaß und die Verschiebung des Problems, je nachdem, welche Indikatoren herangezogen werden. Notwendigerweise wird dabei zwischen Verhalten und Einstellungen unterschieden.

Wenn Menschenfeindlichkeit, Rassismus und Rechtsextremismus, z. B. in Form offener Gewalt oder eben auch im Wahlverhalten zu Tage treten, dann ist dies die Spitze eines Eisberges, dessen Ganzes die historischen Kontinuitäten und gesellschaftlichen Verhältnisse betrifft, die sich nicht zuletzt in den Einstellungen sowie Wert- und Normvorstellungen der Bevölkerung widerspiegeln; mit all deren kognitiven, affektiven und handlungsnahen Komponenten, sodass zwar moderate Zusammenhänge, aber kein Determinismus zwischen abwertenden Einstellungen und diskriminierendem Verhalten besteht (Schütz und Six 1996). Nur, je stärker Einstellungen ausgeprägt sind und auch über die Zeit stabil bleiben, desto enger hängen sie mit Verhalten zusammen (Glasman und Albarracín 2006), was sich insbesondere für emotional verankerte Vorurteile zeigt (Talaska et al. 2008). Dabei variieren feindselige Einstellungen über die Zeit und in unterschiedlichen Teilen der Bevölkerung, sind allerdings bei keiner demografischen Gruppe verhaftet oder allein zu verorten.

Vor dem Hintergrund dieser Beobachtungen und der Frage, wie trennscharf sich Feindseligkeiten und rechtsextreme Orientierungen überhaupt in zunächst demografisch bestimmten Altersgruppen verorten lassen, soll eine differenzierte Analyse von antidemokratischen Orientierungen vorgenommen werden. Ebenso sollen daraus Hinweise auf Fragen der Ursachen von- sowie Bildung und Erziehung gegen Menschenfeindlichkeit und Rechtsextremismus ermittelt werden (Zick et al. 2019a).

Die Analyse soll aus einer interdisziplinär erziehungswissenschaftlichen Perspektive auf die politische Sozialisation junger Heranwachsender bzw. Erwachsener erfolgen, also der jungen Mündigen (Kuhn und Krappmann 2000). Hierfür werden Erkenntnisse der Vorurteilsforschung mit Sozialisationstheorie in Verbindung gebracht. Der Blick ist auf antidemokratische Orientierungen gerichtet, wozu neben rechtsextremen auch rechtspopulistische und menschenfeindliche Einstellungen

\footnotetext{
${ }^{3}$ Auch dass sich der Rechtsextremismus durch das Aussterben von ,Alt-Nazis“ und ,Ewiggestrigen“ erledigen würde (Stöss 1989), verweist eher auf Traditionslinien und Entlastungen (Frei et al. 2019).
} 
gehören. Diese sind im Kern antidemokratisch, weil sie Überzeugungen der Ungleichwertigkeit von Menschen widerspiegeln (Berghan und Zick o.J.). Bevor wir sie genauer definieren, diskutieren wir zunächst Grundüberlegungen zur politischen Sozialisation und gehen dann genauer darauf ein, was hier unter rechtsextremen, rechtspopulistischen wie menschenfeindlichen Überzeugungen verstanden wird. Im Anschluss berichten wir intergenerative Unterschiede in aktuellen Daten einer bevölkerungsrepräsentativen Umfrage. Wir haben dazu Differenzen in den antidemokratischen Orientierungen zwischen Altersgruppen statistisch ausgewertet. Die empirischen Analysen sind auf junge Erwachsene konzentriert, genauer Menschen zwischen 18 und 30 Jahren. Wir analysieren also die politischen Überzeugungen in der späten Adoleszenz im Vergleich zu älteren Altersgruppen der 31- bis 60-Jährigen sowie über 60-Jährigen. Für die Gruppen sind mit Blick auf die Teilhabe an politischen Prozessen unterschiedliche Sozialisationskontexte bedeutsam, die u.a. durch damit einhergehende Macht- und Abhängigkeitsverhältnisse geprägt sind, die sich wiederum in den politischen Orientierungen widerspiegeln können.

Zuletzt werfen wir auch ein Schlaglicht auf einige Faktoren, die neben dem Alter die Variation der Orientierungen in der Bevölkerung erklären können. Hierbei gehen wir heuristisch vor, d. h. wir dokumentieren ausgehend von sich in vorhergegangenen Analysen als erklärungskräftig erwiesenen Einflussfaktoren einige Auffälligkeiten in der empirischen Erklärung rechtsextremer Einstellungen. Der primäre Beitrag gilt aber der Frage nach dem Vorhandensein intergenerativer Unterschiede. Am Ende sollen auf der Grundlage der Analysen auch erste Hinweise für eine demokratische Erziehung und Bildung, die Menschenfeindlichkeit und Rechtsextremismus in der Gesamtbevölkerung ernst nimmt, gegeben werden.

\section{Politische Sozialisation und Orientierungen zur Demokratie}

Eine politische Sozialisation zu demokratischen oder eben antidemokratischen Orientierungen, wie sie sich in Menschenfeindlichkeit, Rechtsextremismus oder anderen politischen Einstellungen manifestieren, ist von den Bedingungen abhängig, die es Individuen überhaupt ermöglichen, ein politisch denkendes und handelndes Subjekt zu werden (vgl. z. B. Rippl et al. 2015). Kleeberg-Niepage (2012, S. 23) betont dies mit dem Blick auf rechtsextremes Denken. Dieses ,[bahne sich] letztlich den gleichen Weg durch die Sozialisationsinstanzen wie demokratisches Denken auch“, was gegen die Idee spricht, „dass Rechtsextremismus eine Sonderform oder eben ,Fehlentwicklung ' politischer Sozialisation darstellt.“

In der politischen Sozialisation setzen sich Menschen aktiv mit ihrer Umwelt auseinander und eignen sich Werte und Normen in Bezug auf das gesellschaftliche Zusammenleben, bzw. dessen Institutionen, Strukturen und Funktionsweisen, sowie Einstellungen zu sozialen Gruppen an. In dieser wechselseitigen Auseinandersetzung der Individuen bzw. Subjekte mit politischer Macht und Ohnmacht besteht ein normativer Gehalt politischer Sozialisation darin, Mitglieder einer demokratischen Gesellschaftsform an eben jenen Verfassungskonsens zu binden (Kevenhörster 2008), der einen gewissen Grad an Unterstützung gegenüber dem politischen 
Systems erfordert, um dessen Stabilität und Legitimität zu gewährleisten (Easton 1975).

Dies entspricht jedoch keiner blinden bzw. unreflektierten Übernahme vorgegebener Werte und Normen. Vielmehr fordern gerade demokratisch verfasste Gesellschaften von ihren Subjekten die selbstständige, produktive, kritische und fortwährende Auseinandersetzung mit der sozialen Wirklichkeit und den gesellschaftlichen Spielregeln. Eine offene und vielfältige Demokratie ist zwar die Voraussetzung für politische Mündigkeit, aber diese Mündigkeit ist ihrerseits Voraussetzung für die Demokratie.

Dafür sind sowohl manifeste und meist formelle Faktoren wie die politische Bildung als auch latente und eher informelle Strukturen und Prozesse relevant. Für die politische Sozialisation und dabei insbesondere die Herausbildung rechtsextremer Einstellung können (früh-)kindliche Bindungs- und Interaktionserfahrungen in der Familie als auch die zunehmende Ablösung von ihr durch deren Bearbeitung im Jugendalter entscheidend sein (Hopf und Hopf 1997; Wahl et al. 2001). Neben Erfahrungen in der Kindheit wird auch die Bedeutung lebenslangen Lernens betont (Preiser 2008). Wobei die Ausbildung einer politischen Identität im Vordergrund steht, die von sozial geteilten Überzeugungen geprägt ist (Adams 1985). Die Adoleszenz ist für die politische Sozialisation eine ,,besonders sensible und prägende Phase“ (Rippl 2008, S. 444), die einerseits Möglichkeiten bietet, eine eigene politische Position einzunehmen, gesellschaftliche Rechte und Pflichten wahrzunehmen, oder auch sich von der gegebenen demokratischen Ordnung abzugrenzen. Mit zunehmenden Alter werden zudem die politischen Orientierungen stabiler (Hooghe und Wilkenfeld 2008) und die Befürwortung demokratischer Werte, Normen und Herrschaftsstrukturen enger miteinander verknüpft (Reinders 2001). Diese Einschätzung wird durch Längsschnittstudien gestützt (Grob 2009). Ebenso ist mit steigendem Alter von einem größeren Einfluss der Sozialisationsinstanzen auf die Ausbildung von Vorurteilen in der Adoleszenz auszugehen, zumal die Forschung zeigt, dass sich nach der Kindheit Vorurteile als identitätsrelevante gruppenbezogene Überzeugungen verfestigen, die junge Menschen an Gruppen binden und ihnen Zugehörigkeit eben in Abgrenzung zu sozial bedeutsamen ,Anderen“ verschaffen (Raabe und Beelmann 2011).

Vor dem Hintergrund der Problemstellung und Erkenntnis, dass antidemokratische Orientierungen frühe Vorläufer in der Entwicklung haben, sich in der Adoleszenz allmählich herausbilden und identitätsstiftend werden können, teilen wir die Ansicht, dass eine Auseinandersetzung mit Menschenfeindlichkeit und Rechtsextremismus im Jugendalter zielgerichtet sein kann oder überhaupt notwendig wird. Allerdings müsste dies, gerade mit einem Blick auf die politische Sozialisation, die von Wechselwirkungen von Personenfaktoren der Identitätsbildung mit der gesellschaftlichen Umwelt ausgeht, die Frage nach dem gesellschaftlichen Klima, den Normen und gesellschaftlichen Ungleichwertigkeitsvorstellungen hervorrufen. Wird nicht danach gefragt, wie Normalitätsvorstellungen der Ungleichwertigkeit von Gruppen prägend sind, können menschenfeindliche und rechtsextreme Sichtweisen im Jugendalter vorschnell als jugendliche Devianz fehlinterpretiert werden. Die Auseinandersetzung mit den Facetten antidemokratischer Orientierungen sollte demgemäß im Verhältnis zu den gesamtgesellschaftlichen Normalitäten, Entwicklungen bzw. Verschiebungen 
und Diskursen kritisch betrachtet werden. Und dazu gehört auch die Analyse von Einstellungen, Überzeugungen und normativen Ungleichwertigkeitsvorstellungen, also Gruppenbezogener Menschenfeindlichkeit, und nicht nur manifesten Straftaten und Organisationsformen.

An dieser Stelle möchten wir die Konzepte politischer bzw. antidemokratischer Orientierungen genauer präziseren. Dies ist notwendig, um deren Messung später zu ermöglichen. Im Kontext der Diskussion sind u. E. menschenfeindliche, rechtsextreme und rechtspopulistische Orientierungen Ausdruck solcher Überzeugungen.

Wir verstehen Gruppenbezogene Menschenfeindlichkeit (GMF) und deren Facetten der Abwertung, wie beispielsweise Rassismus, Antisemitismus oder Sexismus als generalisierte Vorurteile, die zwar jeweils ihre eigene Ausdrucksweise, Geschichte und unterschiedliche Folgen für die Betroffenen haben, die aber nicht unabhängig voneinander bestehen und auf einen gemeinsamen Kern, eine Ideologie der Ungleichwertigkeit, zurückgeführt werden können (vgl. Heitmeyer 2002; Zick et al. 2008, 2019b). ${ }^{4}$ Diese langjährige Beobachtung bestätigt die frühe Annahme, dass eine Person, die Vorurteile beispielsweise in Form rassistischer Meinungen gegenüber einer bestimmten Gruppe hat, mit größerer Wahrscheinlichkeit auch Ungleichwertigkeitsvorstellungen gegenüber anderen ,Fremdgruppen' teilt (Allport 1954).

Diese Gruppen werden auf der Grundlage von Kategorisierungen und Stereotypisierungen gebildet, und zwar in einer Weise, dass sie soziale Hierarchien und Machtverhältnisse in der Gesellschaft ausdrücken wie gleichsam reproduzieren. Entscheidend für die Ausrichtung des sozialen Vorurteils ist kein individuelles Feindschaftsverhältnis, sondern die tatsächliche oder meist vermutete Zugehörigkeit zu einer Gruppe, also unabhängig davon, wie sich eine Person verhält, ob sie sich selbst als Mitglied jener Gruppe sieht oder auch einer anderen Gruppe zuordnet.

Die Gruppenbezogene Menschenfeindlichkeit, die sich in sozialen Vorurteilen manifestiert, kann drei Einstellungsdimensionen umfassen (Aronson et al. 2018). Kognitiv entspricht sie einem sozial geteilten (konstruierten) Wissen über bestimmte Gruppen, das sich z.B. in der Aussage ausdrückt, Sinti und Roma seien kriminell. Affektiv drückt sie sich als emotionale Reaktion wie z.B., dass Homosexualität ekelhaft sei, aus. Drittens können sich Vorurteile verhaltensbezogen, oder direkt im Verhalten ausdrücken. Die Forderung, Muslimen die Einwanderung zu versagen, oder Obdachlose aus Fußgängerzonen zu vertreiben, ist insofern auch Ausdrucks von Vorurteilen bzw. GMF.

Rechtspopulistische Orientierungen greifen GMF auf und binden sie an rechtsradikale aber vermeintlich ,noch normale " und eben scheinbar ,nicht-extremistische " Überzeugungen. Gerade weil rechtspopulistische Orientierungen bislang noch nicht unter einer generativen oder sozialisationsrelevanten Perspektive analysiert wurde, empfiehlt sich eine solche Analyse angesichts der Normalisierungen und Normalisierungseinflüsse des Rechtspopulismus (vgl. aber Bude 1993). Dass rechtspopu-

\footnotetext{
${ }^{4}$ Folgende Elemente werden anknüpfend an die Mitte-Studie 2018/19 als Gruppenbezogene Menschenfeindlichkeit gefasst: Rassismus, Fremdenfeindlichkeit, Antisemitismus, Muslimfeindlichkeit, Abwertung von Sinti und Roma und asylsuchender Menschen, Sexismus, Abwertung homosexueller Menschen, von Trans*Menschen, wohnungsloser Menschen, langzeitarbeitsloser Menschen sowie von Menschen mit Behinderung und die Etabliertenvorrechte. Eine theoretische Definition der einzelnen Elemente findet sich bei Zick et al. (2019b, S. $58 \mathrm{ff}$.).
} 
listische Überzeugungen eine intergenerative Brückenfunktion haben können, zeigt beispielsweise die Ausbildung von Jugendorganisationen oder jugendaffinen rechtsextremen Gruppen, wie z.B. der Identitären Bewegung, oder die intergenerative Verbreitung von Hasskampagnen in digitalen Medien.

Bei unserem Verständnis rechtspopulistischer Einstellungen greifen wir diese sozial geteilten (rassistischen) Vorurteile über vermeintlich fremde oder andere Gruppen auf und verorten sie in einem zweidimensionalen Modell (Mudde und Kaltwasser 2017). Die horizontale Dimension ist geprägt von einem homogenen und damit antipluralistischen Volksverständnis (,Wir'), von dem ,Fremde' ausgeschlossen werden. Die vertikale Dimension ist geprägt von dem Wunsch nach einer Autorität, die härter gegen ,die Anderen' vorzugehen vermag - ausgedrückt in einem Lawand-Order-Autoritarismus -, als auch im Ausdruck eines destruktiven Misstrauens in die Prinzipien der Demokratie und deren Repräsentanten, die allgemein als ,eigennützig' herabgewürdigt werden und das Volk um die Macht betrügen würden (Demokratiemisstrauen bzw. der dezidierten Demokratieverachtung). Im Rechtspopulismus zeigt sich die antipluralistische Idee eines einzig wahren und moralisch reinen Volks in Abgrenzung zu ,den Anderen“. Dieser Populismus ist als ,dünne Ideologie" nicht notwendigerweise extremistisch, genauso wie Rechtsextremismus in seinen verschiedenen Ausprägungen nicht notwendigerweise populistisch ist. Allerdings weisen rechtspopulistische wie rechtsextreme Orientierungen große Nähen und Überschneidungen auf, dies insbesondere in Ideologien der Ungleichwertigkeit, mithin GMF und der Betonung nationaler Identitäten (vgl. auch Zick und Küpper 2018). Mindestens auf einer Einstellungsebene ist darüber hinaus sowohl bei rechtspopulistisch als auch rechtsextrem orientierten Personen eine höhere Billigung und Bereitschaft zu Gewalt nachweisbar (Zick et al. 2019a).

Rechtsextreme Orientierungen haben in Übereinstimmung mit einer Konsensdefinition von Forschenden sechs Einstellungselemente, die zwei Dimensionen zugeordnet werden können (Decker und Brähler 2006; Küpper et al. 2019a). Die Befürwortung einer rechtsgerichteten Diktatur, nationaler Chauvinismus und die Verharmlosung des Nationalsozialismus beziehen sich dabei eher auf eine rechtsextreme Politikvorstellung, die als autoritär, nationalistisch, geschichtsrevisionistisch und explizit antidemokratisch umschrieben werden kann, während Fremdenfeindlichkeit, Antisemitismus und Sozialdarwinismus eher das rechtsextreme Gesellschaftsverständnis kennzeichnen, das von einem rassistischen und völkisch-biologistischen Denken durch die Abwertung von ,Ausländern“, Juden und sozial Schwächeren getragen wird.

Im Folgenden sollen nun die drei antidemokratischen Orientierungen danach untersucht werden, inwieweit sie in Altersgruppen geteilt werden, oder sich tatsächlich eine jugendspezifische oder eher andere Konzentration ergibt. Empirisch werden dabei Einstellungen in der ,Normalbevölkerung ' betrachtet; nicht mehr, aber auch nicht weniger. Die Ergebnisse werden zeigen, welche Ungleichwertigkeitsvorstellungen von wem in welchem Maße geteilt werden und ob die These der besonderen Belastung der Jugend oder die These, dass Ungleichwertigkeitsideologien, die sich womöglich in jugendlichen Hasstaten radikalisieren, auf weit geteilten Einstellungen begründen. 


\section{Intergenerative Differenzen in antidemokratischen Orientierungen in der Mitte-Studie 2018/2019}

\subsection{Datengrundlage und Auswertungsmethode}

Die folgende Analyse basiert in erster Linie auf Querschnittsdaten der Mitte-Studie 2018/195, bei der im Rahmen einer computergestützten Telefonumfrage 2005 Bürger_innen befragt wurden. Da in die Auswertung aus Gründen der Vergleichbarkeit nur diejenigen Befragten einbezogen werden, die eine deutsche Staatsangehörigkeit besitzen, reduziert sich die Stichprobe auf 1890 Befragte. Die Untersuchung 2018/19 beinhaltete einen methodischen Split, der einer zufällig ausgewählten Hälfte der Befragten für Aussagen zur Gruppenbezogenen Menschenfeindlichkeit und einigen weiteren Konstrukten die Möglichkeit gab auf einer vierstufigen vollverbalisierten Likert-Skala zu antworten und der anderen Hälfte auf einer fünfstufigen (Berghan und Faulbaum 2019). Die Items zur Erfassung rechtsextremer Einstellungen wurden durchweg fünfstufig erhoben.

Skalen mit mehr Antwortkategorien spiegeln die tatsächlichen Korrelationen einer normalverteilten kontinuierlichen Variablen besser wider (Bollen und Barb 1981; Lozano et al. 2008; Lubke und Muthén 2009). Der Nachteil des Splits ist die Verringerung der Stichprobengröße, insbesondere, wenn wie im Folgenden Aussagen für spezifische (soziodemografische) Untergruppen getroffen werden sollen. Um dennoch für die Vergleiche zwischen den Altersgruppen auf die Gesamtstichprobe zurückgreifen zu können, wurden für die Ergebnisse für GMF und die Rechtspopulistische Orientierung auf Konstruktebene die 4- und 5-stufigen Antworten zusammengefasst. Dabei wurde die Mittelkategorie „teils-teils“ nicht zur offenen Zustimmung gezählt. Vielmehr wurde für die Analyse in den einzelnen Einstellungs-Dimensionen Dummy-Variablen mit den Ausprägungen ,Zustimmung ' und ,Keine Zustimmung ‘ gebildet. Zur Zustimmung wurden die Antwortkategorien „trifft/stimme eher zu“ sowie ,trifft/stimme voll und ganz zu“ gerechnet. Zur Ablehnung wurde die offene Ablehnung mit ,trifft/stimme eher nicht zu“, ,trifft/stimme überhaupt nicht zu“ sowie die Mittelkategorie „teils-teils“ gezählt. Die Ergebnisse fallen daher eher konservativ aus. Aufgrund dieses Vorgehens werden ordinale Verfahren, insbesondere $\mathrm{Chi}^{2}$ Tests für die Analyse von Unterschieden im intergenerativen Vergleich verwendet. Lediglich für die ergänzend berichtete lineare Regressionsanalyse der Einflussfaktoren rechtsextremer Einstellungen in den verschiedenen Altersgruppen sowie die Testung der internen Konsistenz und Faktorenstruktur der Messinstrumente ${ }^{6}$ wur-

\footnotetext{
5 Die Mitte-Studie wurde durch Prof. Dr. Andreas Zick am Institut für interdisziplinäre Konflikt- und Gewaltforschung an der Universität Bielefeld geleitet und gefördert durch die Friedrich-Ebert-Stiftung, Berlin.

6 Alle Messinstrumente weisen im Vergleich über die Altersgruppen hinweg eine ausreichende interne Konsistenz auf. Ursprünglich war eine Analyse der Abwertung von Menschen mit Behinderung geplant, allerdings korrelieren die beiden diesbezüglichen Aussagen in der jüngsten Altersgruppe nicht und das Messinstrument weißt in der jüngsten Altersgruppe keine interne Konsistenz auf. Wir haben uns daher entschieden, die Ergebnisse des intergenerativen Vergleichs für dieses GMF-Element nicht zu berichten. Das Messinstrument wurde in der aktuellsten Erhebung der Mitte-Studie verändert (vgl. Zick et al. 2019b, S. 74f.) und es scheint für zukünftige Erhebungen eine weitere Verbesserung der Messung notwendig.
} 
de auf entsprechende Analyseverfahren zurückgegriffen, dann jedoch lediglich mit fünfstufig erhobenen Skalen gerechnet.

Das Durchschnittsalter beträgt 51,4 Jahre (die/der jüngste Befragte war 18 Jahre, die/der älteste 97 Jahre alt); mit 52,2\% sind etwas mehr weibliche als männliche Befragte in der Stichprobe vertreten. Für den Vergleich der rechtsextremen Einstellungen im Zeitverlauf wird zusätzlich auf Daten der Mitte-Studien aus den Jahren 2014 (Zick und Klein 2014) sowie 2016 (Zick et al. 2016) zurückgegriffen. Die rechtsextremen Einstellungen sind auf dieselbe Art und Weise erhoben worden, wie 2018/19. Alle Daten sind mit einem kombinierten Gewicht aus Alter und Bildung gewichtet. Näheres zur Methodik und Stichprobe der jeweiligen Erhebungen findet sich bei Groß (2014), Krause und Faulbaum (2016) sowie Berghan und Faulbaum (2019).

Die im GMF-Konzept eingegangenen Abwertungselemente werden jeweils über Kurzskalen mit zwei vorurteilsvollen und diskriminierenden Aussagen gemessen, die sich zum Monitoring menschenfeindlicher Einstellungen in Deutschland etabliert haben (Heitmeyer 2002; Krause und Zick 2013; Zick et al. 2008, 2019b). Für die Operationalisierung rechtspopulistischer Einstellungen orientieren wir uns an der Methode in der Mitte-Studie (Küpper et al. 2019b). Für die horizontale Konfliktdimensionen werden die vier GMF-Dimensionen herangezogen, die im Zentrum rechtspopulistischer Agitation stehen: Fremden- und Muslimfeindlichkeit, Abwertung von Sinti und Roma sowie von Asylsuchenden. Wenngleich weitere Formen von GMF, wie beispielsweise Antisemitismus oder ein klassisch-biologistischer Rassismus, wesentlicher Teil des Rechtspopulismus sein können, fließen sie aus Gründen der Vergleichbarkeit nicht in den Index ein. Für die vertikale Konfliktdimension beziehen wir die Konstrukte des Demokratiemisstrauens und einer autoritären Aggression ein. Insgesamt fließen damit sechs Einstellungselemente in den Gesamtindex einer rechtspopulistischen Orientierung ein (Küpper et al. 2019b). ${ }^{7}$ Als klar rechtspopulistisch eingestellt und damit im Zustimmungsbereich des Gesamtindex Rechtspopulismus gelten jene Befragte deren Wert über alle Dimensionen hinweg im Durchschnitt $\geq 3$ (bei vierstufiger Erhebung) beziehungsweise $\geq 3,5$ bei fünfstufiger Erhebung liegt. Demgegenüber wird jedes der sechs Elemente des Rechtsextremismus anknüpfend an die aufgeführte Konsensdefinition und die Operationalisierung in der Mitte-Studie mit drei Aussagen erfasst, welche auf einer fünfstufigen vollverbalisierten Antwortskala von „lehne völlig ab“ bis ,stimme voll und ganz zu“ zur Beantwortung stehen (Küpper et al. 2019a). Analog zu Küpper et al. (2019a) sowie Decker et al. (2012) wird ein Summenindex gebildet, um ein rechtsextremes Weltbild zu erfassen. Bei insgesamt 18 Items (d.h. bei einem minimalen Wert von 18 und maximalen Wert von 90) gilt eine Person ab einem Grenzwert von >63 als

\footnotetext{
7 Das Messmodell wurde mittels Strukturgleichungsmodell von Küpper et al. (2019b) geprüft (vgl. S. 184) und es bestätigt empirisch die theoretische Struktur eines latenten Faktors zweiter Ordnung „Rechtspopulismus" hinter den im Text genannten Faktoren erster Ordnung. Die interne Konsistenz des Gesamtindex Rechtspopulismus ist in allen Altersgruppen hoch. Bei jüngster Altersgruppe $\alpha=0,88$; bei mittlerer $\alpha=0,87$ und bei ältester $\alpha=0,88$. Über alle Altersgruppen hinweg $\alpha=0,88$. Die Faktorenstruktur wurde mittels Hautachsenanalyse mit oblimin Rotation getrennt nach den drei Altersgruppen geprüft und die Interpretation der Faktorenanalyse lässt nicht auf relevante Unterschiede zwischen den Altersgruppen schlieBen.
} 
geschlossen rechtsextrem eingestellt. Alle Erhebungsinstrumente sind in Tab. 3 im Anhang aufgeführt.

In der Stichprobe der Jahre 2014 und 2016 waren die jüngsten Befragten 16 Jahre alt. Wir haben diese nicht aus der jahresvergleichenden Analyse ausgeschlossen, um die jüngste Altersgruppe bestmöglich abbilden zu können, und schließlich soll ein maximal möglicher intergenerativer Vergleich vorgenommen werden. Es ist anzumerken, dass sich bei einer Einteilung in kleinere Altersgruppen zwar die Differenzierbarkeit zwischen den Altersgruppen erhöht, allerdings liegen unserer Analyse Messinstrumente extremer Einstellungen zugrunde, bei denen teilweise nur ein relativ geringer Teil der Bevölkerung zustimmt. Wenn statt drei zwischen sechs verschiedenen Altersgruppen differenziert wird, fällt in einigen Gruppen die Fallzahl im Zustimmungsbereich so gering aus, dass insbesondere zu einzelnen Facetten rechtsextremer Einstellungen nicht mehr ausreichend robuste Aussagen hinsichtlich der Altersunterschiede zwischen den Gruppen getroffen werden können. Nichtsdestotrotz versuchen wir, die in dieser Analyse berichteten Ergebnisse auch hinsichtlich ihrer Replizierbarkeit in kleiner eingeteilten Altersgruppen zu prüfen, insbesondere für GMF und Rechtspopulismus. ${ }^{8}$ In der Regel kommen die so durchgeführten Analysen nicht zu grundsätzlich anderen Ergebnissen, einzelne relevante Auffälligkeiten diesbezüglich werden bei der Ergebnisdarstellung transparent gemacht.

\subsection{Ergebnisse}

Im Folgenden beschreiben wir die Ergebnisse und Auffälligkeiten der deskriptiven Analyse. Zunächst konzentriert sich der Beitrag auf die primäre Forschungsfrage und damit auf eine Exploration intergenerativer Unterschiede in den verschiedenen Einstellungsfacetten in der aktuellsten Erhebung 2018/19. Daran anschließend werden die rechtsextremen Orientierungen untersucht und Auffälligkeiten in der Entwicklung der Zustimmung der jüngeren Altersgruppe im Vergleich zu den anderen Altersgruppen seit 2014 dargelegt. Im Anschluss werden zentrale Einflussfaktoren rechtsextremer Einstellungen untersucht, um erste Hinweise auf mögliche Ursachen zu erkennen. Diese Analyse kann nur verkürzt erfolgen und ist nicht hinreichend, weil die Querschnittdaten keine kausalen Analysen zulassen. Sie geben aber Hinweise auf die Frage, warum die Orientierungen variieren und weisen die Richtung für weitere vertiefende Studien.

Abb. 1 zeigt, dass bei sieben Elementen ein deutlicher und signifikanter Anstieg in der Zustimmung zur Abwertung mit dem Lebensalter erkennbar ist: Besonders beispielsweise beim Sexismus $\left(\chi^{2}(2)=22,93 ; p=0,000 ; \mathrm{V}\right.$ (Cramér's V) $=0,11$ ), wo sich die Zustimmung in der jüngsten Altersgruppe gegenüber der ältesten Altersgruppe nahezu vervierfacht oder in den Etabliertenvorrechten $\left(\chi^{2}(2)=30,65\right.$;

\footnotetext{
${ }^{8}$ Die Kontrolle mit sechs Alterskategorien bietet sich nicht für die Einstellungsdimensionen an, bei denen nur eine sehr geringe Anzahl an Befragten im Zustimmungsbereich liegt, da die Anzahl in den einzelnen soziodemografischen Gruppen zu gering ist. Insbesondere bei den besonders hart formulierten Aussagen zum Rechtsextremismus ist dies der Fall. Diese wurden zwar mit sechs Alterskategorien überprüft und die Ergebnisse unterscheiden sich in Ihren Aussagen nicht relevant voneinander, jedoch ist die Fallzahl im Rechtsextremismus in einigen Gruppen so gering, dass eine Auswertung aller sechs Alterskategorien keine robusten Aussagen erlaubt und aus diesem Grund nicht berichtet wird.
} 


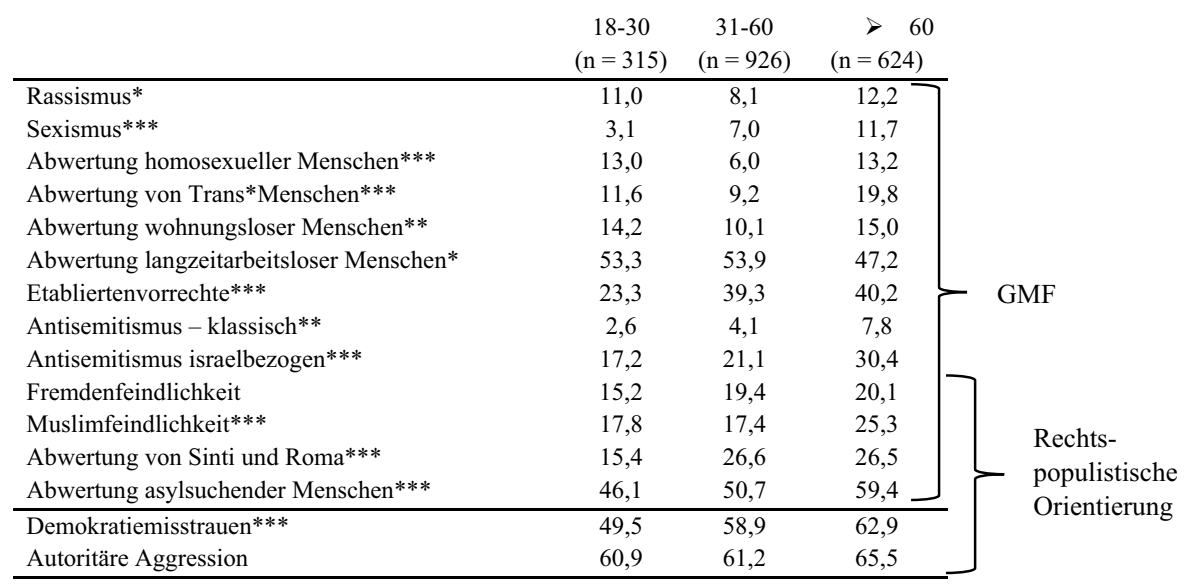

Abb. 1 Zustimmung (\%) zu den Facetten Gruppenbezogener Menschenfeindlichkeit und rechtspopulistischer Einstellungen in verschiedenen Altersgruppen

$p=0,000 ; \mathrm{V}=0,13)$, bei denen sich die Zustimmung gegenüber der mittleren und ältesten Altersgruppe fast verdoppelt. Auch im klassischen $\left(\chi^{2}(2)=14,51 ; p=0,001\right.$; $\mathrm{V}=0,09)$ und israelbezogenen Antisemitismus $\left(\chi^{2}(2)=24,37 ; p=0,000 ; \mathrm{V}=0,12\right)$, der Muslimfeindlichkeit $\left(\chi^{2}(2)=15,24 ; p=0,000 ; \mathrm{V}=0,09\right)$, der Abwertung von Sinti und Roma $\left(\chi^{2}(2)=17,24 ; p=0,000 ; \mathrm{V}=0,10\right)$, sowie von asylsuchenden Menschen $\left(\chi^{2}(2)=18,56 ; p=0,000 ; V=0,10\right)$ zeigt sich der Anstieg menschenfeindlicher Vorurteile mit dem Lebensalter recht deutlich. Bei der Fremdenfeindlichkeit $\left(\chi^{2}(2)=3,22 ; p=0,200 ; \mathrm{V}=0,04\right)$ zeigt sich ein Anstieg der Zustimmung mit dem Lebensalter zwar ebenfalls, jedoch ist dieser statistisch nicht signifikant und daher nur in der Tendenz beobachtbar.

Bei vier Elementen zeigen sich signifikante Unterschiede zwischen den Altersgruppen, die sich eher einer U-förmige Verteilung annähern, d.h. bei der sich die geringste Zustimmung zu GMF in der mittleren Altersgruppe erkennen lässt. Dies ist im Rassismus ( $\left.\chi^{2}(2)=7,40 ; p=0,025 ; \mathrm{V}=0,06\right)$, der Abwertung homosexueller Menschen $\left(\chi^{2}(2)=27,19 ; p=0,000 ; \mathrm{V}=0,12\right)$, von Trans*Menschen $\left(\chi^{2}(2)=36,66 ; p=0,000 ; \mathrm{V}=0,14\right)$ sowie der Abwertung von wohnungslosen Menschen $\left(\chi^{2}(2)=9,38 ; p=0,009 ; \mathrm{V}=0,07\right)$ der Fall. Auf den zweiten Blick zeigt sich jedoch auch hier, dass es nicht die jüngste Altersgruppe ist, die die höchste Menschenfeindlichkeit aufweist. Bei einer Einteilung der Altersgruppen in sechs Kategorien mit einer jüngsten Altersgruppe zwischen 18-27 Jahren zeigt diese Gruppe niedrigere Werte im Rassismus als die nächst ältere Gruppe. Eine quasi U-Förmige Verteilung bleibt jedoch mit Blick auf die weiteren Altersgruppen und die höhere Zustimmung der Ältesten bestehen $\left(\chi^{2}(5)=15,78 ; p=0,007 ; \mathrm{V}=0,09\right) .{ }^{9}$ Ähnlich verhält es sich bei der Abwertung homosexueller Menschen $\left(\chi^{2}(5)=42,42\right.$;

\footnotetext{
9 6,2\% der jüngsten Altersgruppe (bis 27 Jahre) stimmt beim Rassismus zu, gegenüber 12,1\% (28-37); 7,6\% (38-47); 9,9\% (48-57); 7,7\% (58-67); 13,9\% (ab 68 Jahre).
} 
$p=0,000 ; \mathrm{V}=0,15) .{ }^{10}$ Bei der Abwertung von Trans*Menschen sind die Unterschiede zwischen jüngster und mittlerer Altersgruppe vernachlässigbar. Die U-Form zeigt sich nur in der Tendenz, denn die älteste Altersgruppe stimmt mit 19,8\% rund doppelt so häufig zu wie die anderen Jahrgänge. Bleibt die Abwertung wohnungsloser Menschen, bei der sich unter Kontrolle der Ergebnisse mit sechs Alterskategorien $\left(\chi^{2}(5)=17,08 ; p=0,004 ; V=0,10\right)$ ebenfalls zeigt, dass die 28- bis 37-Jährigen mit $14,5 \%$ eine höhere Zustimmung haben als die 18 - bis 27 -Jährigen $(9,1 \%)$. Der Trend der niedrigen Zustimmung in den mittleren Altersgruppen und der höchsten Zustimmung bei den Ältesten bleibt ansonsten auch bei sechs Alterskategorien bestehen. Es sind jedoch nicht die jüngsten Befragten, welche für eine U-förmige Verteilung der Ergebnisse sorgen, sondern die Befragten im Alter ab 28 Jahren bis Mitte/Ende Dreißig sowie die älteste Altersgruppe.

Die Abwertung langzeitarbeitsloser Menschen zeigt als einziges GMF-Element eine signifikante Abnahme der Zustimmungen zu den vorurteilsvollen Meinungen gegenüber der Gruppe mit dem Lebensalter (53,3\% Zustimmung bei 18- bis 30Jährigen vs. $47,2 \%$ bei über 60 -Jährigen) $\left(\chi^{2}(2)=7,13 ; p=0,028 ; V=0,06\right)$. Die Unterschiede sind jedoch nicht besonders groß und auch die Kontrolle mit sechs Alterskategorien relativiert diese. ${ }^{11}$ Die Abnahme in der Zustimmung zur Abwertung Langzeitarbeitsloser geht nicht von den jüngsten (18-27) aus - diese weisen vielmehr gemeinsam mit den Altersgruppen ab 58 Jahren die niedrigsten Zustimmungen auf, sondern die höchste Zustimmung zeigt sich bei den 28- bis 37- sowie 38- bis 47-Jährigen. Nichtsdestotrotz zeigt sich auch bei Kontrolle mit sechs Alterskategorien das im Vergleich zu den anderen GMF Elementen auffallende Ergebnis, dass die ältesten Personen(gruppen) die niedrigste Zustimmung aufweisen.

Mit Blick auf die für die Erfassung einer rechtspopulistischen Orientierung herangezogenen Konstrukte des Demokratiemisstrauen und der autoritären Aggression zeigen sich mit den GMF-Ergebnissen vergleichbare Tendenzen (siehe Abb. 1). Für das Demokratiemisstrauen ist ein signifikanter Anstieg in der Zustimmung mit dem Lebensalter feststellbar $\left(\chi^{2}(2)=15,30 ; p=0,000 ; \mathrm{V}=0,09\right)$. Die älteste Altersgruppe stimmt deutlich häufiger zu als die jüngste Altersgruppe. Bei Kontrolle mit sechs Alterskategorien bleibt die Zunahme im Demokratiemisstrauen mit dem Lebensalter bestehen ${ }^{12}$, jedoch weist die jüngste Altersgruppe bis 27 Jahren deutlich höhere Zustimmung auf als die 28- bis 37-Jährigen, welche stattdessen die niedrigste Zustimmung zeigen. Für die autoritäre Aggression zeigt sich nur ein tendenzieller Anstieg mit dem Lebensalter, der jedoch statistisch nicht signifikant ist $\left(\chi^{2}(2)=3,38\right.$; $p=0,185 ; \mathrm{V}=0,04)$. Bei Kontrolle mit sechs Alterskategorien zeigt sich im Autoritarismus ein signifikanter Unterschied, der jedoch keinen linearen Anstieg wiedergibt. Vielmehr zeigt sich die niedrigste Zustimmung bei den jüngsten sowie den 48- bis

\footnotetext{
$107,8 \%$ der jüngsten Altersgruppe (bis 27 Jahre) stimmt bei der Abwertung Homosexueller zu, gegenüber $13,8 \%$ (28-37); 5,1\% (38-47); 5,3\% (48-57); 6,8\% (58-67); $16 \%$ (ab 68 Jahre).

11 18-27: 48,5\% Zustimmung; 28-37: 57,3\%; 38-47: 57,5\%; 48-57: 53,7\%; 58-67: 46,6\%; >67 Jahre: $47,1 \%$. $\left(\chi^{2}(5)=15,35 ; p=0,000 ; \mathrm{V}=0,09\right)$.

12 18-27: 57,1\% Zustimmung; 28-37: 46,6\%; 38-47: 56,9\%; 48-57: 61,1\%; 58-67: 65,8\%; >67 Jahre: $61,2 \% .\left(\chi^{2}(5)=26,02 ; p=0,000 ; \mathrm{V}=0,12\right)$.
} 
Tab. 1 Zustimmung (\%) zu den Dimensionen Rechtsextremer Einstellungen in verschiedenen Altersgruppen in 2014, 2016 und 2018/19

\begin{tabular}{|c|c|c|c|c|c|c|c|c|c|}
\hline & \multicolumn{3}{|l|}{$16 / 18-30$} & \multicolumn{3}{|l|}{$31-60$} & \multicolumn{3}{|l|}{$>60$} \\
\hline & $\begin{array}{l}2014 \\
(n=379)\end{array}$ & $\begin{array}{l}2016 \\
(n=353)\end{array}$ & $\begin{array}{l}2018 / 19 \\
(n=315)\end{array}$ & $\begin{array}{l}2014 \\
(n=905)\end{array}$ & $\begin{array}{l}2016 \\
(n=918)\end{array}$ & $\begin{array}{l}2018 / 19 \\
(n=926)\end{array}$ & $\begin{array}{l}2014 \\
(n=601)\end{array}$ & $\begin{array}{l}2016 \\
(n=605)\end{array}$ & $\begin{array}{l}2018 / 19 \\
(n=624)\end{array}$ \\
\hline $\begin{array}{l}\text { Befürwortung } \\
\text { rechtsautori- } \\
\text { tärer Diktatur }\end{array}$ & 4,9 & 5,1 & 2,2 & 3,7 & 3,2 & 4,8 & 4,2 & 3,2 & 1,9 \\
\hline Chauvinismus & 14,7 & 7,5 & 12,9 & 9,0 & 12,4 & 12,6 & 15,3 & 15,8 & 12,4 \\
\hline $\begin{array}{l}\text { Verharmlosung } \\
\text { des National- } \\
\text { sozialismus }\end{array}$ & 3,5 & 1,9 & 7,0 & 0,9 & 2,2 & 1,5 & 2,2 & 1,9 & 1,7 \\
\hline $\begin{array}{l}\text { Fremden- } \\
\text { feindlichkeit }\end{array}$ & 8,3 & 2,2 & 6,2 & 5,9 & 8,2 & 9,5 & 9,3 & 9,8 & 9,8 \\
\hline $\begin{array}{l}\text { Anti- } \\
\text { semitismus }\end{array}$ & 2,9 & 1,0 & 4,7 & 2,4 & 1,7 & 2,6 & 4,8 & 4,6 & 4,3 \\
\hline $\begin{array}{l}\text { Sozial- } \\
\text { darwinismus }\end{array}$ & 1,1 & 2,1 & 4,9 & 1,2 & 1,5 & 2,0 & 3,6 & 2,7 & 1,1 \\
\hline
\end{tabular}

67-Jährigen, die höchste hingegen bei den 28- bis 47- sowie den über 68-Jährigen. ${ }^{13}$ Ausgehend von den berichteten Ergebnissen überrascht jedoch insgesamt nicht, dass sich auch für den Gesamtindex Rechtspopulismus (Tab. 2), in den vier GMF-Elemente, welche die Abwertung von als fremd konstruierten sowie das Demokratiemisstrauen und die autoritäre Aggression einfließen, sich demgemäß eine höhere Zustimmung in den älteren Altersgruppe zeigt $\left(\chi^{2}(2)=7,37 ; p=0,025 ; \mathrm{V}=0,06\right)$. Dies ändert sich auch nicht bei Kontrolle mit sechs Alterskategorien $\left(\chi^{2}(5)=12,14\right.$; $p=0,033 ; \mathrm{V}=0,08)$.

Bei der Analyse rechtsextremer Einstellungen in der Erhebung 2018/19 fällt auf, dass sich für drei der sechs Dimensionen des Rechtsextremismus signifikante Unterschiede zwischen den drei Altersgruppen feststellen lassen. Die Ergebnisse sind in Tab. 1 abgedruckt. Die mittlere Altersgruppe stimmt verhältnismäßig am stärksten einer Diktatur zu, während sich die jüngste und älteste kaum voneinander unterschei$\operatorname{den}\left(\chi^{2}(2)=11,14 ; p=0,004 ; V=0,08\right)$. Besonders auffallend sind die signifikanten Unterschiede in den Zustimmungen zur Verharmlosung des Nationalsozialismus $\left(\chi^{2}(2)=30,70 ; p=0,000 ; V=0,14\right)$ sowie zum Sozialdarwinismus $\left(\chi^{2}(2)=14,65\right.$; $p=0,001 ; \mathrm{V}=0,09)$, welche der Beobachtung eines Anstiegs antidemokratischer Einstellungen wie in den meisten menschenfeindlichen Vorurteilen mit dem Lebensalter nicht entsprechen. Bei der Verharmlosung des Nationalsozialismus liegen in der jüngsten Altersgruppe $7 \%$ der Befragten im Zustimmungsbereich, während dies bei den Mittleren nur 1,5\% beziehungsweise bei den über 60-Jährigen 1,7\% sind. Ähnlich verhält es sich beim Sozialdarwinismus. Mit Blick auf den Gesamtindex Rechtsextremismus (Tab. 2) zeigt sich in der Tendenz ein höherer Anteil von jüngeren Befragten im Zustimmungsbereich, allerdings sind diese Unterschiede statistisch nicht signifikant $\left(\chi^{2}(2)=3,39 ; p=0,183 ; \mathrm{V}=0,05\right)$.

13 18-27: 58,4\% Zustimmung; 28-37: 65,2\%; 38-47: 65,1\%; 48-57: 57,2\%; 58-67: 57,3\%; >67 Jahre: $69,7 \% \cdot\left(\chi^{2}(5)=20,33 ; p=0,001 ; \mathrm{V}=0,11\right)$ 
Tab. 2 Verbreitung manifest rechtsextremer und rechtspopulistischer Orientierungen (Zustimmung auf den Gesamtindexen in \%) in verschiedenen Altersgruppen

\begin{tabular}{llll}
\hline & $\begin{array}{l}18-30 \\
(n=315)\end{array}$ & $\begin{array}{l}31-60 \\
(n=926)\end{array}$ & $\begin{array}{l}>60 \\
(n=624)\end{array}$ \\
\hline Rechtspopulistische Orientierung* & 16,4 & 21,5 & 24,1 \\
Rechtsextreme Orientierung & 4,1 & 2,0 & 2,1 \\
\hline
\end{tabular}

Ausgehend von diesen Auffälligkeiten in rechtsextremen Orientierungen scheint es von Relevanz, insbesondere die Zustimmung zur rechtsextremen Einstellungen in den Altersgruppen hinsichtlich ihrer Entwicklung in den letzten Jahren in den Blick zu nehmen (Tab. 1). ${ }^{14}$ Werden die Veränderungen oder Stabilitäten in dem Ausmaß des Rechtsextremismus in den Altersgruppe genauer betrachtet, fällt zunächst auf, dass es in der Gruppe der älteren Befragten nur geringe Veränderungen und insbesondere keinen Anstieg in der Zustimmung seit 2014 gibt. In der Verharmlosung des Nationalsozialismus $\left(\chi^{2}(2)=0,58 ; p=0,750 ; \mathrm{V}=0,02\right)$, dem Antisemitismus $\left(\chi^{2}(2)=0,19 ; p=0,911 ; \mathrm{V}=0,01\right)$, der Fremdenfeindlichkeit $\left(\chi^{2}(2)=0,10 ; p=0,950 ; \mathrm{V}=0,01\right)$ zur Befürwortung einer Diktatur $\left(\chi^{2}(2)=5,44\right.$; $p=0,066 ; \mathrm{V}=0,06)$ und dem Chauvinismus $\left(\chi^{2}(2)=2,95 ; p=0,228 ; \mathrm{V}=0,04\right)$ zeigen sich keine signifikanten Unterschiede im Analysezeitraum. Nur beim Sozialdarwinismus $\left(\chi^{2}(2)=8,33 ; p=0,015 ; \mathrm{V}=0,07\right)$ zeigt sich ein Rückgang in der Zustimmung der älteren Befragten seit 2014. Etwas anders verhält es sich in der mittleren Altersgruppe. Keine der Dimensionen rechtsextremer Orientierung ist rückläufig. Vielmehr zeigt sich über nahezu alle Dimensionen hinweg mindestens in der Tendenz ein Anstieg zwischen 2014 und 2018/19. Statistisch signifikant wird dies jedoch nur beim Chauvinismus $\left(\chi^{2}(2)=7,33 ; p=0,026 ; \mathrm{V}=0,05\right)$ und der Fremdenfeindlichkeit $\left(\chi^{2}(2)=8,47 ; p=0,014 ; V=0,06\right) .{ }^{15}$ Anders verhält es sich in der jüngsten Altersgruppe. Während die Befürwortung einer Diktatur (2014: 4,9\%; 2016: $5,1 \% ; 2018 / 19: 2,2 \%)\left(\chi^{2}(2)=4,31 ; p=0,116 ; \mathrm{V}=0,07\right)$ keine statistisch signifikante Veränderung aufweist, so zeigt sich bei den restlichen Dimensionen eher ein sehr aktueller Anstieg in der Zustimmung, nachdem diese 2016 im Vergleich zu 2014 zunächst zurückgegangen war. Dies wird insbesondere im Chauvinismus $\left(\chi^{2}(2)=9,27 ; p=0,010 ; \mathrm{V}=0,10\right)$ und bei der Verharmlosung des Nationalsozialis-

\footnotetext{
${ }^{14}$ Wir stellen den Vergleich über die Jahre aus zwei Gründen für den Zeitraum ab 2014 an. Zum einen aus einem methodischen Grund, da für diesen Zeitraum auf Daten zurückgriffen werden kann, bei denen der Rechtsextremismus mit gleichbleibender Methode erhoben wurde. Zum anderen weil die Entwicklung rechtsextremer Einstellungen in den verschiedenen Altersgruppen im Zuge der neueren Flüchtlingsbewegungen und der zugenommenen Aufnahme von Geflüchteten in Deutschland, dem damit einhergehenden gesellschaftlichen Diskurs sowie dem Aufstieg der AfD und weiterer rechtsextremer Akteure in Deutschland in diesem Zeitraum von Interesse sind. Wir verzichten auf eine gesonderte Analyse der GMF und Rechtspopulismusdimensionen ab 2014, da hierfür im Sinne der Parsimonität zum einen der Raum in diesem Beitrag fehlt, die Erhebungsinstrumente zum Rechtsextremismus auch zentrale menschenfeindliche Facetten beinhalten und uns insbesondere die berichteten Auffälligkeiten im Rechtsextremismus bemerkenswert erscheinen und größeres Analysepotenzial beinhalten.

15 Keine signifikanten Unterschiede in der mittleren Altersgruppe zwischen 2014, 2016 und 18/19 bei Befürwortung einer Diktatur $\left(\chi^{2}(2)=3,16 ; p=0,206 ; \mathrm{V}=0,04\right)$; Verharmlosung des $\mathrm{NS}\left(\chi^{2}(2)=4,89\right.$; $p=0,087 ; \mathrm{V}=0,05)$; Antisemitismus $\left(\chi^{2}(2)=1,82 ; p=0,403 ; \mathrm{V}=0,03\right)$; Sozialdarwinismus $\left(\chi^{2}(2)=1,90\right.$; $p=0,386 ; \mathrm{V}=0,03)$.
} 
mus $\left(\chi^{2}(2)=11,68 ; p=0,003 ; \mathrm{V}=0,11\right)$ deutlich. Aber auch bei der Fremdenfeindlichkeit $\left(\chi^{2}(2)=13,43 ; p=0,001 ; V=0,11\right)$ und im Antisemitismus $\left(\chi^{2}(2)=7,45\right.$; $p=0,024 ; \mathrm{V}=0,09)$. Der Sozialdarwinismus steigt in der jüngsten Altersgruppe seit 2014 mehr oder wenig kontinuierlich $\operatorname{an}\left(\chi^{2}(2)=10,30 ; p=0,006 ; \mathrm{V}=0,10\right)$.

An dieser Stelle drängt sich die Frage auf, ob Einflussfaktoren die Variation in den berichteten Einstellungen erklären können. Dazu haben wir in Übereinstimmung mit theoretischen Ausführungen und empirischen Analysen von Zick und Küpper (2018) bzw. Küpper et al. (2019c) den Gesamtindex rechtsextremer Orientierung empirisch unter Berücksichtigung von zentralen und gut erforschten demografischen, soziologischen und sozialpsychologischen Faktoren erklärt. Dazu zählen, die Soziale Dominanzorientierung, autoritäre Aggression, Anomie, politische Machtlosigkeit, ökonomistische Werthaltung, fraternale Deprivation (gefühlter Mangel an Ressourcen im Vergleich zu ,Fremden“), Gerechtigkeitsgefühle, sowie die demografischen Faktoren Geschlecht und formales Bildungsniveau. Dafür haben wir in den drei Altersgruppen separate Regressionsanalysen durchgeführt, deren Ergebnisse in den Tab. 4, 5, 6 und 7 im Anhang aufgeführt sind. Es zeigt sich: In allen drei Altersgruppen haben die Erklärungsfaktoren ähnliche Einflüsse. Einen auffälligen Unterschied gibt es jedoch im Bildungseffekt auf rechtsextreme Einstellungen. Während unter Kontrolle der genannten Erklärungsfaktoren der Einfluss des formalen Bildungsniveaus in der mittleren und ältesten Altersgruppe verschwindet, bleibt er in der jüngsten Altersgruppe erklärungsstark.

\section{Diskussion}

Ausgangspunkt der empirischen Analysen war die Frage, ob die Lokalisierung und Problematisierung von antidemokratischen Orientierungen insbesondere unter jungen Menschen angemessen ist, angesichts eines zunächst augenscheinlich klaren Ausmaßes an Hasstaten oder öffentlich sichtbaren Gruppen junger Rechtsextremist_innen sowie der politischen Konzentration von pädagogischer Praxis auf junge Menschen. Wir haben eine empirische Antwort gegeben. Jüngere Befragte stimmen zwar einzelnen Dimensionen rechtsextremer Einstellungen deutlich häufiger zu, aber die älteren Befragtengruppen sind in gewisser Weise ,normal feindselig ' ${ }^{16}$ und äußern hinsichtlich Gruppenbezogener Menschenfeindlichkeit im Durchschnitt die höchsten Zustimmungen. Damit scheint sich der klassische Befund höherer feindseliger Einstellungen mit steigendem Lebensalter teils zu bestätigen, jedoch ist der Anstieg autoritärer Einstellungen nicht eindeutig linear. Dies könnte daran liegen, dass wir lediglich die autoritäre Aggression gemessen haben und nicht tiefergehende autoritär bis kulturell-konservative Einstellungen, die mit einer höheren Menschenfeindlichkeit bei älteren Gruppen in Verbindung gebracht werden (Franssen et al. 2013).

\footnotetext{
16 Die Formulierung ,normal feindselig“ hebt auf die langjährige Beobachtung ab, dass Vorurteile sich gesamtgesellschaftlich und individuell so verbreiten und verfestigen können, dass sie als Normalität und vermeintliche Tatsachen wahrgenommen werden, die sich wiederum selbst aufrechterhalten und legitimieren (vgl. Zick und Küpper 2006).
} 
Das ist anders in Bezug auf rechtsextreme Orientierungen. Sie finden deutlicher Zuspruch bei den Jüngeren.

Aber die Frage, wer am Ende antidemokratischer eingestellt ist, verkürzt vorab die Risiken und Herausforderungen an eine politische Sozialisation zur Demokratie. Demokratische Gesellschaften sind nicht durch die Überzeugungen einzelner Generationen gefährdet, denn Facetten antidemokratischer Ideologien der Ungleichwertigkeit sind empirisch in allen Altersgruppen nachweisbar. Das Ausmaß variiert und bedarf auch einer Einschätzung, ab wann einzelne Versatzstücke oder Auffälligkeiten in Teilen der Bevölkerung, gefährlich` bzw. antidemokratisch sind. Ebenso ist die Frage zu stellen, welche konsistenten Muster und Strukturen der Abwertung und Ausgrenzung auf Mechanismen der Selbstentlastung hinweisen. Auch darauf geben die Auswertungen erste Hinweise. Eine sich als demokratisch und offen verstehende Mitte der Gesellschaft, die Menschenfeindlichkeit, Rechtspopulismus und Rechtsextremismus auf die bedrohlichen Ränder projiziert, läuft Gefahr, eigene Bezüge auf Ideologien der Ungleichwertigkeit zu normalisieren und legitimieren sowie die politische Dimension des eigenen Handelns nicht ernst zu nehmen. Fuchs (2003) konstatiert, dass die ,Verdächtigung ' der Jugend, eher anfällig für Rechtsextremismus zu sein, eine Verengung eines Diskurses ist auf einen unter Umständen „sichtbare[n] Teil entsprechender Einstellungen und Handlungsdispositionen, die weit darüber hinaus in der Bevölkerung latent vorhanden sind“" (S. 655). Durch die Selbstentlastung fungieren Jugendphänomene aber als gesellschaftliche Stellvertreter für ein politisches Klima der Abwertung, Ausgrenzung und Gewalt (Groffmann 2001).

Mit Blick auf die ergänzende Ursachenanalyse und die dabei festgestellte Auffälligkeit des unterschiedlichen Bildungseinflusses auf Rechtsextremismus je nach Alter ist zunächst festzuhalten, dass tatsächlich stattfindende Bildungsprozesse über das formale Bildungsniveau nur unzureichend operationalisiert werden können. Dennoch wird deutlich, dass Bildung insbesondere bei der jüngeren Altersgruppe einen schwächenden Einfluss auf die Zustimmung zu einem rechtsextremen Weltbild hat. Zwei Erklärungen dafür erscheinen uns diskussionswürdig. ${ }^{17}$ Zum einen könnte ein Kohorteneffekt vorliegen. Bildungsprozesse in Schulen hatten vor einigen Jahrzehnten evtl. nicht in gleichem Maße demokratiefördernde Wirkung wie in jüngerer Zeit. Es ist vorstellbar, dass jüngere Kohorten in Bildungsinstitutionen sozialisiert werden, die Demokratie und die damit verbundenen Werte eher erfahrbar machen, während ältere Kohorten noch stärker autoritäreren Bildungsinstitutionen ausgesetzt waren, die weniger politische bzw. demokratiefördernde Bildung vermittelten. Zum anderen könnte auch ein Lebensphasen- bzw. Alterseffekt vorliegen, d.h., dass Bildung zwar im Allgemeinen eine vorurteilsmindernde oder demokratiefördernde Wirkung hat, dies jedoch bei Jüngeren am ehesten noch einen Effekt zeigt, da sie sich noch in Bildungsinstitutionen befinden bzw. deren Einfluss eher noch nachwirken. Demgegenüber sind Ältere mit größerer Wahrscheinlichkeit in anderen politischen So-

\footnotetext{
17 Wir können an dieser Stelle keine Erklärungen für den allgemein klassischen Befund eines inversen Zusammenhangs zwischen formalem Bildungsniveau und Vorurteilen (sog. Bildungseffekt) aufführen, sondern verweisen auf diesbezügliche Arbeiten (u. a. Heyder 2003; Hopf 1999; Hughes 2017; Ostapczuk et al. 2008).
} 
zialisationskontexten eingebettet, die keine genuin liberalisierende Wirkung haben und die durchlebten Bildungserfahrungen werden in den Lebenskontexten älterer Menschen weniger relevant. Die Prüfung beider Annahmen ist ein Forschungsdesiderat und kann mit den hier geprüften Daten nicht beantwortet werden. Obwohl diese ergänzenden Ergebnisse nicht detaillierter ausgeführt werden können, haben wir sie betrachtet, da sie die deskriptiven Analyse bestätigen. Es kann angemessen sein, die Analyse und Diskussion sowie eine daraus abgeleitete Prävention von antidemokratischen Orientierungen auf eine bestimmte Altersspanne auszurichten, wenn längsschnittliche Entwicklungen darauf hinweisen, dass Einstellungsanteile in den betreffenden Bevölkerungsgruppen konstant bleiben oder deutlich zunehmen. Allerdings weisen sowohl das Vorkommen verschiedener antidemokratischer Orientierungen in allen Altersgruppen als auch die nahezu gleichen Erklärungskräfte rechtsextremer Orientierung in allen Altersgruppen umso mehr auf die Notwendigkeit einer gesamtgesellschaftlichen Betrachtungsweise des Phänomens hin. Es ist angebracht, das Alter bzw. die Adoleszenz als vorrangigen Erklärungsfaktor für Rechtsextremismus und Menschenfeindlichkeit weiter in Frage zu stellen (vgl. auch Dierbach 2010). ${ }^{18}$

Stattdessen gilt es wissenschaftlich zu analysieren und pädagogisch zu reflektieren, wie sich Ungleichwertigkeit in verschiedensten Facetten in gesamtgesellschaftlichen Diskursen und Praktiken manifestiert. Denn die mal mehr, mal weniger subtil geteilte Menschenfeindlichkeit bietet die Legitimationsfolie, vor der ein aktionsorientierter Rechtsextremismus erst schlagkräftig wird. So ist insbesondere hinsichtlich der aufgeführten auffälligen Veränderungen im Rechtsextremismus der jüngeren Befragten seit $2014 \mathrm{zu}$ bedenken, dass soziale Einstellungen immer eingebettet sind in gesellschaftliche Entwicklungen. Die politische Sozialisation jüngerer Generationen ist stets abhängig von gesellschaftlich relevanten sozialen Ordnungen und Ideologien insbesondere hinsichtlich der Beurteilung von Gruppen. Schenke et al. (2018) gehen im Rahmen von Fokusgruppeninterviews mit 16- bis 35-Jährigen der Frage nach, ob das Auftreten von Gruppierungen wie Pegida in den letzten Jahren Spuren in der politischen Kultur hinterlassen hat. Sie kommen zwar zu dem Ergebnis, dass es keine direkten Anzeichen für ein Anwachsen antidemokratischen Potenzials gegeben hat, jedoch auch keine deutliche Distanzierung zu den völkisch-rassistischen und rechtspopulistischen Inhalten unter jungen Menschen.

Die Dominanz gesellschaftlicher Entwicklungen könnte hinsichtlich der auffälligen Zustimmung jüngerer Befragter zum Sozialdarwinismus als Zuspitzung einer gewissen Leistungs- und Nützlichkeitslogik interpretiert werden, der sich unter 30Jährige in ihrer beruflichen und sozialen Lebenslage womöglich selbst besonders ausgesetzt sehen. So verweist auch die Shell Jugendstudie darauf, dass Jugendliche „,bereit [sind] sich in hohem Maße an Leistungsnormen zu orientieren“ (Albert et al. 2019, S. 13) Der Sozialdarwinismus ist die Schattenseite einer rigiden, teils auch ökonomistischen Leistungsorientierung und eines sozialen Kontexts, der den Wert

18 In Tab. 4 ist darüber hinaus ersichtlich, dass der Alterseffekt auf rechtsextreme Einstellungen verschwindet, wenn die genannten Konstrukte in die Analyse einbezogen werden. Die verwendeten Erklärungsfaktoren sind also relevant zur Erklärung des Alterseffekts, steigen jedoch in Ihrer Erklärungskraft darüber hinaus. Hieran sollte in zukünftigen Analysen angeschlossen werden. 
von Menschen nach deren Nützlichkeit bestimmt. Auch andere Studien stellen fest, markorientierte und ökonomistische Einstellungen bedingen Menschenfeindlichkeit und Gewalt (Groß und Hövermann 2014; Groß et al. 2018; Hövermann et al. 2015).

Mit Blick auf die relativ hohe Zustimmung der Jüngeren zur Verharmlosung des Nationalsozialismus greifen wir die Einschätzung von Küpper et al. (2019a) auf. Es scheint eine Distanz der jüngeren Befragten zum Nationalsozialismus zu herrschen, welcher als historischer Effekt interpretiert werden könnte, den Bildung bisher nicht in ausreichendem Maße ausgleichen konnte; anders ausgedrückt: „Die Jüngeren verlieren die historische und persönliche Nähe zum Nationalsozialismus“ (S. 143). Auch die Ergebnisse des Erinnerungsmonitor „MeMO“ lassen solche Schlüsse zu (Zick et al. 2018). Es könnte aber auch sein, dass die andauernde Agitation insbesondere von AfD-Anhänger_innen und weiterer neurechter Akteure hinsichtlich der Relativierung der Bedeutsamkeit des Nationalsozialismus für die deutsche Geschichte oder die Forderung die Erinnerungskultur zu ändern, einen bleibenden Eindruck insbesondere auf jene (jüngeren) Teile der Bevölkerung hinterlassen hat, welche in ihren politischen Einstellungen noch nicht so verfestigt sind, wie ältere Generationen. Dies würde auch die relativ geringen Veränderungen in den rechtsextremen Einstellungsfacetten bei älteren Befragten seit 2014 erklären. In den letzten Jahren waren etwas größere Veränderungen in der mittleren Altersgruppe und insgesamt die auffälligsten Veränderungen in der jüngsten Altersgruppe festzustellen, was auf Veränderungen des gesellschaftlichen Klimas hinweist. „Allerdings ist die Forschung in diesem Feld vielmehr als in anderen Bereichen gezwungen, ihre einmal gewonnenen Ergebnisse kritisch zu hinterfragen - von Generation zu Generation verändert sich die Kohorte der Jugendlichen hinsichtlich ihre Wertvorstellungen und Einstellungen“" (Langebach 2016, S. 419).

Die Frage nach der pädagogischen Praxis, die der Analyse folgen sollte, drängt sich auf. Wenngleich konkrete praktisch pädagogische Vorschläge einzig aufgrund unserer Ergebnisse kaum gegeben werden können, so lässt die Analyse doch Hinweise auf eine gewisse Perspektive zu, die pädagogische Praxis einnehmen kann. Welche Erziehung und Bildung hat die Ermittlung antidemokratischer Orientierung zur Folge? Demokratiepädagogik, politische Bildung und andere Ansätze, die sich den hier analysierten Phänomenen annehmen, stehen vor großen Herausforderungen angesichts dessen, dass (junge) Antidemokrat_innen sich auf ein umfangreiches Reservoire von menschenfeindlichen, rechtspopulistischen und rechtsextremen Orientierungen in der Mitte der Gesellschaft berufen können. Die präsentierten Ergebnisse lassen allerdings etwas Zuversicht zu. Bildung und Erziehung üben einen Einfluss auf die Entwicklung von menschenfeindlichen bis rechtsextremen Orientierungen aus und es dürfte sich lohnen, wenn sich Bildungsanstrengungen nicht nur auf die hauptsächlich in formale Bildungsinstitutionen eingebundene jüngere Altersgruppe konzentrieren. Die Entwicklung von politischen Sozialisations- und Bildungsangeboten über die gesamte Lebensspanne ist angebracht, selbst unter der Annahme, dass das Jugendalter eine sensible Phase für die Herausbildung und Verfestigung ebenjener Orientierungen ist. Doch dies steht eben nicht vor einem politisch-kulturell oder diskursiv leeren Sozialisationshintergrund. Erziehung und Bildung sehen sich daher maßgeblich vor der Herausforderung stets in Auseinandersetzung mit „der überwältigenden Kraft des Bestehenden“ (Adorno 1982 [1969]) die sozialen 
Zustände zu reflektieren und auch eine gewisse Widerständigkeit gegenüber dem normal feindlichen einzuüben und zu vermitteln.

Funding Open Access funding enabled and organized by Projekt DEAL.

Open Access Dieser Artikel wird unter der Creative Commons Namensnennung 4.0 International Lizenz veröffentlicht, welche die Nutzung, Vervielfältigung, Bearbeitung, Verbreitung und Wiedergabe in jeglichem Medium und Format erlaubt, sofern Sie den/die ursprünglichen Autor(en) und die Quelle ordnungsgemäß nennen, einen Link zur Creative Commons Lizenz beifügen und angeben, ob Änderungen vorgenommen wurden.

Die in diesem Artikel enthaltenen Bilder und sonstiges Drittmaterial unterliegen ebenfalls der genannten Creative Commons Lizenz, sofern sich aus der Abbildungslegende nichts anderes ergibt. Sofern das betreffende Material nicht unter der genannten Creative Commons Lizenz steht und die betreffende Handlung nicht nach gesetzlichen Vorschriften erlaubt ist, ist für die oben aufgeführten Weiterverwendungen des Materials die Einwilligung des jeweiligen Rechteinhabers einzuholen.

Weitere Details zur Lizenz entnehmen Sie bitte der Lizenzinformation auf http://creativecommons.org/ licenses/by/4.0/deed.de. 


\section{Anhang}

Tab. 3 Aussagen zur Erfassung gruppenbezogener Menschenfeindlichkeit, rechtspopulistischer und rechtsextremer Einstellungen (Angabe der Mittelwerte, Standardabweichung und Reliabilität der Skalen)

\section{Gruppenbezogene Menschenfeindlichkeit}

Rassismus

(4er-Split: $\mathrm{M}=1,54 ; \mathrm{SD}=0,70 ; n=911 ; \alpha=0,42$ । 5er-Split: $\mathrm{M}=1,87 ; \mathrm{SD}=1,08 ; n=970 ; \alpha=0,61$ )

Aussiedler sollten besser gestellt sein als Ausländer, da sie deutscher Abstammung sind

Die Weisen sind zu Recht führend in der Welt

Fremdenfeindlichkeit

(4er-Split: $\mathrm{M}=1,86 ; \mathrm{SD}=0,94 ; n=910 ; \alpha=0,76$ | 5er-Split: $\mathrm{M}=2,19 ; \mathrm{SD}=1,19 ; n=972 ; \alpha=0,74$ )

Es leben zu viele Ausländer in Deutschland

Wenn Arbeitsplatze knapp werden, sollte man die in Deutschland lebenden Ausländer wieder in ihre Heimat zurückschicken

\section{Muslimfeindlichkeit}

(4er-Split: $\mathrm{M}=1,87 ; \mathrm{SD}=0,92 ; n=904 ; \alpha=0,68$ । 5er-Split: $\mathrm{M}=2,30 ; \mathrm{SD}=1,26 ; n=958 ; \alpha=0,74$ )

Durch die vielen Muslime hier fühle ich mich manchmal wie ein Fremder im eigenen Land

Muslimen sollte die Zuwanderung nach Deutschland untersagt werden

Abwertung asylsuchender Menschen

(4er-Split: $\mathrm{M}=2,80 ; \mathrm{SD}=0,87 ; n=905 ; \alpha=0,54$ । 5er-Split: $\mathrm{M}=3,39 ; \mathrm{SD}=1,05 ; n=972 ; \alpha=0,52$ )

Bei der Prüfung von Asylantragen sollte der Staat großzügig sein

Die meisten Asylbewerber werden in ihrem Heimatland gar nicht verfolgt

Abwertung von Sinti und Roma

(4er-Split: $\mathrm{M}=2,04 ; \mathrm{SD}=0,95 ; n=898 ; \alpha=0,82$ । 5er-Split: $\mathrm{M}=2,37 ; \mathrm{SD}=1,22 ; n=965 ; \alpha=0,81$ )

Ich hatte Probleme damit, wenn sich Sinti und Roma in meiner Gegend aufhalten

Sinti und Roma neigen zu Kriminalität

Antisemitismus - klassisch

(4er-Split: $\mathrm{M}=1,31 ; \mathrm{SD}=0,65 ; n=877 ; \alpha=0,76$ । 5er-Split: $\mathrm{M}=1,46 ; \mathrm{SD}=0,85 ; n=939 ; \alpha=0,78$ )

Juden haben in Deutschland zu viel Einfluss

Durch ihr Verhalten sind Juden an ihren Verfolgungen mitschuldig

Antisemitismus - israelbezogen

(4er-Split: $\mathrm{M}=1,31 ; \mathrm{SD}=0,65 ; n=877 ; \alpha=0,68$ । 5er-Split: $\mathrm{M}=2,40 ; \mathrm{SD}=1,19 ; n=905 ; \alpha=0,65$ )

Bei der Politik, die Israel macht, kann ich gut verstehen, dass man etwas gegen Juden hat

Was der Staat Israel heute mit den Palästinensern macht, ist im Prinzip auch nichts Anderes als das, was die Nazis im Dritten Reich mit den Juden gemacht haben

Sexismus

(4er-Split: $\mathrm{M}=1,41 ; \mathrm{SD}=0,73 ; n=914 ; \alpha=0,79$ | 5er-Split: $\mathrm{M}=1,61 ; \mathrm{SD}=0,96 ; n=976 ; \alpha=0,74$ )

Für eine Frau sollte es wichtiger sein, ihrem Mann bei seiner Karriere zu helfen, als selbst Karriere zu machen

Frauen sollten sich wieder mehr auf die Rolle der Ehefrau und Mutter besinnen

Abwertung homosexueller Menschen

(4er-Split: $\mathrm{M}=1,39 ; \mathrm{SD}=0,76 ; n=905 ; \alpha=0,78$ । 5er-Split: $\mathrm{M}=1,71 ; \mathrm{SD}=1,81 ; n=965 ; \alpha=0,83$ )

Es ist ekelhaft, wenn Homosexuelle sich in der Öffentlichkeit küssen

Homosexualität ist unmoralisch

Abwertung von Trans*Menschen

(4er-Split: $\mathrm{M}=1,55 ; \mathrm{SD}=0,88 ; n=907 ; \alpha=0,76$ । 5er-Split: $\mathrm{M}=1,87 ; \mathrm{SD}=1,21 ; n=964 ; \alpha=0,77$ )

Ich finde es albern, wenn ein Mann lieber eine Frau sein will oder umgekehrt, eine Frau lieber ein Mann

Transsexuelle und Transgender sollten versuchen, nicht so aufzufallen 
Tab. 3 (Fortsetzung)

Abwertung wohnungsloser Menschen

(4er-Split: $\mathrm{M}=1,70 ; \mathrm{SD}=0,76 ; n=908 ; \alpha=0,53$ । 5er-Split: $\mathrm{M}=2,15 ; \mathrm{SD}=1,11 ; n=968 ; \alpha=0,61$ )

Die meisten Obdachlosen sind arbeitsscheu

Bettelnde Obdachlose sollten aus den Fußgängerzonen entfernt werden

Abwertung langzeitarbeitsloser Menschen

(4er-Split: $\mathrm{M}=2,72 ; \mathrm{SD}=0,99 ; n=909 ; \alpha=0,80$ । 5er-Split: $\mathrm{M}=3,24 ; \mathrm{SD}=1,28 ; n=964 ; \alpha=0,80$ )

Die meisten Langzeitarbeitslosen sind nicht wirklich daran interessiert, einen Job zu finden

Ich finde es empörend, wenn sich die Langzeitarbeitslosen auf Kosten der Gesellschaft ein bequemes

Leben machen

\section{Etabliertenvorrechte}

(4er-Split: $\mathrm{M}=2,51 ; \mathrm{SD}=0,95 ; n=910 ; \alpha=0,57$ । 5er-Split: $\mathrm{M}=2,90 ; \mathrm{SD}=1,21 ; n=976 ; \alpha=0,62$ )

Wer irgendwo neu ist, sollte sich erst mal mit weniger zufrieden geben

Wer schon immer hier lebt, sollte mehr Rechte haben, als die, die später zugezogen sind

\section{Rechtspopulistische Einstellungen}

\section{Rechtsgerichteter Autoritarismus}

(4er-Split: $\mathrm{M}=2,96 ; \mathrm{SD}=0,96 ; N=910 ; \alpha=0,76$ । 5er-Split: $\mathrm{M}=3,48 ; \mathrm{SD}=1,26 ; N=972 ; \alpha=0,81$ )

Verbrechen sollten härter bestraft werden

Um Recht und Ordnung zu bewahren, sollte man härter gegen Außenseiter und Unruhestifter vorgehen

Demokratiemisstrauen

(4er-Split: $\mathrm{M}=2,82 ; \mathrm{SD}=0,85 ; N=914 ; \alpha=0,77$ | 5er-Split: $\mathrm{M}=3,37 ; \mathrm{SD}=1,06 ; N=977 ; \alpha=0,78$ )

Die demokratischen Parteien zerreden alles und lösen die Probleme nicht

Politiker umgehen die bestehenden Gesetze, wenn es um ihre eigenen Vorteile geht

Politiker nehmen sich mehr Rechte heraus als normale Bürger

\section{Rechtsextreme Einstellungen}

Befürwortung einer rechtsgerichteten Diktatur

$(\mathrm{M}=5,12 ; \mathrm{SD}=2,81 ; n=1825 ; \alpha=0,69)$

Im nationalen Interesse ist unter bestimmten Umständen eine Diktatur die bessere Staatsform

Was Deutschland jetzt braucht, ist eine einzige starke Partei, die die Volksgemeinschaft insgesamt verkörpert

Wir sollten einen Führer haben, der Deutschland zum Wohle aller mit starker Hand regiert

Chauvinismus

$(\mathrm{M}=7,30 ; \mathrm{SD}=3,39 ; n=1763 ; \alpha=0,78)$

Wir sollten endlich wieder Mut zu einem starken Nationalgefühl haben

Was unser Land heute braucht, ist ein hartes und energisches Durchsetzen deutscher Interessen gegenüber dem Ausland

Das oberste Ziel der deutschen Politik sollte es sein, Deutschland die Macht und Geltung zu verschaffen, die ihm zusteht

Verharmlosung des Nationalsozialismus

$(\mathrm{M}=4,77 ; \mathrm{SD}=2,58 ; n=1685 ; \alpha=0,72)$

Ohne Judenvernichtung würde man Hitler heute als großen Staatsmann ansehen

Die Verbrechen des Nationalsozialismus sind in der Geschichtsschreibung weit übertrieben worden

Der Nationalsozialismus hatte auch seine guten Seiten 
Tab. 3 (Fortsetzung)

\section{Fremdenfeindlichkeit}

$(\mathrm{M}=6,29 ; \mathrm{SD}=3,25 ; n=1818 ; \alpha=0,82)$

Die Ausländer kommen nur hierher, um unseren Sozialstaat auszunutzen

Wenn Arbeitsplatze knapp werden, sollte man die Ausländer wieder in ihre Heimat zurückschicken

Die Bundesrepublik ist durch die vielen Ausländer in einem gefährlichen Maß überfremdet

Antisemitismus

$(\mathrm{M}=4,56 ; \mathrm{SD}=2,60 ; n=1579 ; \alpha=0,86)$

Auch heute noch ist der Einfluss der Juden zu groß

Die Juden arbeiten mehr als andere Menschen mit üblen Tricks, um das zu erreichen, was sie wollen

Die Juden haben einfach etwas Besonderes und Eigentümliches an sich und passen nicht so recht zu uns

Sozialdarwinismus

$(\mathrm{M}=4,71 ; \mathrm{SD}=2,55 ; n=1811 ; \alpha=0,72)$

Wie in der Natur sollte sich in der Gesellschaft immer der Stärkere durchsetzen

Eigentlich sind die Deutschen anderen Völkern von Natur aus überlegen

Es gibt wertvolles und unwertes Leben

Tab. 4 Erklärung rechtsextremer Einstellungen durch theoriebasierte Prädiktoren

\begin{tabular}{|c|c|c|c|c|}
\hline Prädiktor & Modell 1 & Modell 2 & Modell 3 & Modell 4 \\
\hline$\overline{\text { Alter }}$ & $\begin{array}{l}-0,12(0,02) \\
* * *\end{array}$ & $\begin{array}{l}-0,05(0,02) \\
\text { n.s. }\end{array}$ & $\begin{array}{l}-0,03(0,02) \\
\text { n.s. }\end{array}$ & $\begin{array}{l}-0,01(0,02) \\
\text { n.s. }\end{array}$ \\
\hline Geschlecht & $\begin{array}{l}-0,02(0,71) \\
\text { n.s. }\end{array}$ & $\begin{array}{l}-0,09(0,83) \\
* *\end{array}$ & $\begin{array}{l}-0,08(0,72) \\
* *\end{array}$ & $\begin{array}{l}-0,07(0,73) \\
* *\end{array}$ \\
\hline Formales Bildungsniveau & $\begin{array}{l}-0,33(0,44) \\
* * *\end{array}$ & $\begin{array}{l}-0,18(0,56) \\
* * *\end{array}$ & $\begin{array}{l}-0,11(0,50) \\
* * *\end{array}$ & $\begin{array}{l}-0,10(0,51) \\
* *\end{array}$ \\
\hline $\begin{array}{l}\text { Soziale Dominanzorien- } \\
\text { tierung }\end{array}$ & - & $\begin{array}{l}0,32(0,51) \\
* * *\end{array}$ & $\begin{array}{l}0,23(0,46) \\
* * *\end{array}$ & $\begin{array}{l}0,23(0,47) \\
* * *\end{array}$ \\
\hline AUT-Aggression & - & $\begin{array}{l}0,35(0,35) \\
* * *\end{array}$ & $\begin{array}{l}0,17(0,33) \\
* * *\end{array}$ & $\begin{array}{l}0,16(0,33) \\
* * *\end{array}$ \\
\hline Anomie & - & - & $\begin{array}{l}0,38(0,36) \\
* * *\end{array}$ & $\begin{array}{l}0,35(0,38) \\
* * *\end{array}$ \\
\hline Politische Machtlosigkeit & - & - & $0,08(0,35) * *$ & $0,06(0,36) *$ \\
\hline Ökonomist. Werthaltung & - & - & $\begin{array}{l}0,12(0,44) \\
* * *\end{array}$ & $\begin{array}{l}0,10(0,45) \\
* * *\end{array}$ \\
\hline Fraternale Deprivation & - & - & - & $0,09(0,42) * *$ \\
\hline Gerechtigkeitsgefühl & - & - & - & $\begin{array}{l}-0,09(0,32) \\
* *\end{array}$ \\
\hline $\begin{array}{l}\text { Erklärte Varianz (ange- } \\
\text { passtes } \mathrm{R}^{2} \text { ) }\end{array}$ & $9,9 \%$ & $39,4 \%$ & $54,8 \%$ & $56,6 \%$ \\
\hline
\end{tabular}

Blockweise lineare Regressionsanalyse

nur fünfstufig erhobene Daten

standardisierte Beta-Koeffizienten, Standardfehler (in Klammern) und Signifikanzen 
Tab. 5 Erklärung rechtsextremer Einstellungen in der Altersgruppe 18-30 Jahre durch theoriebasierte Prädiktoren

\begin{tabular}{ll}
\hline Prädiktor & Modell bei 18- bis 30-Jährigen \\
\hline Geschlecht & $-0,08(1,54)$ n.s. \\
Formales Bildungsniveau & $-0,28(1,01) * * *$ \\
Soziale Dominanzorientierung & $0,28(1,07) * * *$ \\
Autoritäre Aggression & $0,17(0,64) * *$ \\
Anomie & $0,32(0,94) * * *$ \\
Politische Machtlosigkeit & $-0,08(0,84)$ n.s. \\
Ökonom. Werthaltung & $-0,03(0,92)$ n.s. \\
Fraternale Deprivation & $0,03(0,88)$ n.s. \\
Gerechtigkeitsgefühl & $-0,17(0,66) * *$ \\
Erklärte Varianz (angepasstes $\left.\mathrm{R}^{2}\right)$ & $71,9 \%$ \\
Fallzahl $(N)$ & 148 \\
\hline
\end{tabular}

Lineare Regressionsanalyse

nur fünfstufig erhobene Daten

standardisierte Beta-Koeffizienten, Standardfehler (in Klammern) und Signifikanzen

Tab. 6 Erklärung rechtsextremer Einstellungen in der Altersgruppe 31-60 Jahre durch theoriebasierte Prädiktoren

\begin{tabular}{ll}
\hline Prädiktor & Modell bei 31- bis 60-Jährigen \\
\hline Geschlecht & $-0,06(1,11) \mathrm{n} . \mathrm{s}$. \\
Formales Bildungsniveau & $-0,02(0,82) \mathrm{n} . \mathrm{s}$. \\
Soziale Dominanzorientierung & $0,20(0,65) * * *$ \\
Autoritäre Aggression & $0,16(0,51) * *$ \\
Anomie & $0,31(0,55) * * *$ \\
Politische Machtlosigkeit & $0,12(0,52) * *$ \\
Ökonomistisch Werthaltung & $0,16(0,67) * * *$ \\
Fraternale Deprivation & $0,08(0,60) \mathrm{n} . \mathrm{s}$. \\
Gerechtigkeitsgefühl & $-0,14(0,48) * *$ \\
Erklärte Varianz (angepasstes $\left.\mathrm{R}^{2}\right)$ & $50,7 \%$ \\
Fallzahl $(N)$ & 345 \\
\hline
\end{tabular}

Lineare Regressionsanalyse nur fünfstufig erhobene Daten standardisierte Beta-Koeffizienten, Standardfehler (in Klammern) und Signifikanzen 
Tab. 7 Erklärung rechtsextremer Einstellungen in der Altersgruppe über 60 Jahre durch theoriebasierte Prädiktoren

\begin{tabular}{ll}
\hline Prädiktor & Modell bei über 60-Jährigen \\
\hline Geschlecht & $0,01(1,23) \mathrm{n} . \mathrm{s}$. \\
Formales Bildungsniveau & $-0,08(0,82) \mathrm{n} . \mathrm{s.}$ \\
Soziale Dominanzorientierung & $0,18(0,90) * *$ \\
Autoritäre Aggression & $0,18(0,58) * *$ \\
Anomie & $0,48(0,62) * * *$ \\
Politische Machtlosigkeit & $0,03(0,58) \mathrm{n} . \mathrm{s}$. \\
Ökonom. Werthaltung & $0,12(0,82) *$ \\
Fraternale Deprivation & $0,16(0,78) * *$ \\
Gerechtigkeitsgefühl & $0,06(0,59) \mathrm{n} . \mathrm{s}$. \\
Erklärte Varianz (angepasstes $\left.\mathrm{R}^{2}\right)$ & $62,0 \%$ \\
Fallzahl $(N)$ & 177 \\
\hline
\end{tabular}

Lineare Regressionsanalyse

nur fünfstufig erhobene Daten

standardisierte Beta-Koeffizienten, Standartfehler (in Klammern) und Signifikanzen

\section{Literatur}

Adams, G. R. (1985). Identity and political socialization. New Directions for Child and Adolescent Development. https://doi.org/10.1002/cd.23219853006.

Adorno, T.W. (1982). Erziehung zur Mündigkeit. In G. Kadelbach (Hrsg.), Erziehung zur Mündigkeit (8. Aufl., S. 140-155). Frankfurt a. M.: Suhrkamp.

Albert, M., Hurrelmann, K., \& Quenzel, G. (2019). Jugend. Eine Generation meldet sich zu Wort (18. Shell Jugendstudie). Weinheim: Beltz.

Allport, G. W. (1954). The nature of prejudice. Cambridge: Perseus Books.

Aronson, E., Wilson, T. D., Akert, R. M., \& Sommers, S. R. (2018). Social psychology (9. Aufl.). Columbus: Pearson.

Baier, D., Pfeiffer, C., Simonson, J., \& Rabold, S. (2009). Jugendliche in Deutschland als Opfer und Täter von Gewalt. Erster Forschungsbericht zum gemeinsamen Forschungsprojekt des Bundesministeriums des Innern und des KFN (Forschungsbericht Nr. 107). Hannover: Kriminologisches Forschungsinstitut Niedersachsen e. V. (KFN).

Berghan, W., \& Faulbaum, F. (2019). Methodik und Design der Mitte-Studie 2018/19. In A. Zick, B. Küpper \& W. Berghan (Hrsg.), Verlorene Mitte - Feindselige Zustände. Rechtsextreme Einstellungen in Deutschland 2018/19 (S. 41-51). Bonn: Dietz.

Berghan, W., \& Zick, A (o. J.). Antidemocratic prejudice-the empirical impact of attitudes toward democracy on group-focused enmity. under review

Bollen, K. A., \& Barb, K. H. (1981). Pearson's R and Coarsley categorized measures. American Sociological Review, 46(2), 232-239.

Bude, H. (1993). Eine abgewehrte soziale Bewegung? Der jugendliche Rechtspopulismus in der neuen Bundesrepublik. Merkur: Deutsche Zeitschrift für europäisches Denken, 47(5), 444-449.

Bundesministerium des Innern (2016). Verfassungsschutzbericht 2015. https://www.verfassungsschutz.de/ embed/vsbericht-2015.pdf. Zugegriffen: 23. Okt. 2019.

Bundesministerium für Familie, Senioren, Frauen und Jugend. (2016). Strategie der Bundesregierung zur Extremismusprävention und Demokratieförderung. https://www.bmfsfj.de/blob/109002/ 5278d578ff8c59a19d4bef9fe4c034d8/strategie-der-bundesregierung-zur-extremismuspraeventionund-demokratiefoerderung-data.pdf. Zugegriffen: 22. Okt. 2019.

Decker, O., \& Brähler, E. (2006). Vom Rand zur Mitte. Rechtsextreme Einstellungen und ihre Einflussfaktoren in Deutschland. Berlin: Friedrich-Ebert-Stiftung.

Decker, O., Kiess, J., \& Brähler, E. (2012). Die Mitte im Umbruch. Rechtsextreme Einstellungen in Deutschland 2012 (herausgegeben für die Friedrich-Ebert-Stiftung von Ralf Melzer). Bonn: Dietz. 
Dierbach, S. (2010). Jung - rechts - unpolitisch? Die Ausblendung des Politischen im Diskurs über rechte Gewalt (Reihe Theorie Bilden, Bd. 19). Bielefeld: transcript.

Easton, D. (1975). A re-assessment of the concept of political support. British Journal of Political Science, $5(4), 435-457$.

Endrikat, K. (2006). Jüngere Menschen. Größere Ängste, geringere Feindseligkeit. In W. Heitmeyer (Hrsg.), Deutsche Zustände (Folge 4, S. 101-114). Frankfurt a. M.: Suhrkamp.

Forschungsgruppe Wahlen (2019a). Landtagswahl in Brandenburg. 01. September 2019. https://www. forschungsgruppe.de/Wahlen/Wahlanalysen/Newsl_Bran190902.pdf. Zugegriffen: 14. Jan. 2020.

Forschungsgruppe Wahlen (2019b). Landtagswahl in Sachsen. 01. September 2019. https://www. forschungsgruppe.de/Wahlen/Wahlanalysen/Newsl_Sach190903.pdf. Zugegriffen: 14. Jan. 2020.

Forschungsgruppe Wahlen (2019c). Landtagswahl in Thüringen. 27. Oktober 2019. https://www. forschungsgruppe.de/Wahlen/Wahlanalysen/Newsl_Thue191108.pdf. Zugegriffen: 14. Jan. 2020.

Franssen, V., Dhont, K., \& van Hiel, A. (2013). Age-related differences in ethnic prejudice: evidence of the mediating effect of right-wing attitudes. Journal of Community \& Applied Social Psychology, 23(3), 252-257.

Frei, N., Maubach, F., Morina, C., \& Tändler, M. (2019). Zur rechten Zeit. Wider die Rückkehr des Nationalismus. Berlin: Ullstein.

Frindte, W., Geschke, D., Haußecker, N., \& Schmidtke, F. (2016). Ein systematisierender Überblick über Entwicklungslinien der Rechtsextremismusforschung von 1990 bis 2013. In W. Frindte, D. Geschke, N. Haußecker \& F. Schmidtke (Hrsg.), Rechtsextremismus und „Nationalsozialistischer Untergrund“. Interdisziplinäre Debatten, Befunde und Bilanzen (Edition Rechtsextremismus, S. 25-98). Wiesbaden: Springer VS.

Fuchs, M. (2003). Rechtsextremismus von Jugendlichen. Zur Erklärungskraft verschiedener theoretischer Konzepte. Kölner Zeitschrift für Soziologie und Sozialpsychologie, 55(4), 654-678.

Glasman, L.R., \& Albarracín, D. (2006). Forming attitudes that predict future behaviour: a meta-analysis of the attitude-behaviour relation. Psychological Bulletin, 132(5), 778-822.

Grob, U. (2009). Die Entwicklung politischer Orientierungen vom Jugend- ins Erwachsenenalter - Ist die Jugend eine spezifisch sensible Phase in der politischen Sozialisation? In H. Fend, F. Berger \& U. Grob (Hrsg.), Lebensverläufe, Lebensbewältigung, Lebensglück. Ergebnisse der LifE-Studie (S. 329-372). Wiesbaden: VS.

Groffmann, A. C. (2001). Das unvollendete Drama. Jugend- und Skinheadgruppen im Vereinigungsprozess (Forschung Soziologie, Bd. 129). Opladen: Leske + Budrich.

Groß, E. M. (2014). Untersuchungsanlage, Methodik und Stichprobe der Analysen. In A. Zick \& A. Klein (Hrsg.), Fragile Mitte - Feindselige Zustände. Rechtsextreme Einstellungen in Deutschland 2014 (S. 24-31, herausgegeben für die Friedrich-Ebert-Stiftung von Ralf Melzer). Bonn: Dietz.

Groß, E. M., \& Hövermann, A. (2014). Marktförmiger Extremismus - ein Phänomen der Mitte? In A. Zick \& A. Klein (Hrsg.), Fragile Mitte - Feindselige Zustände. Rechtsextreme Einstellungen in Deutschland 2014 (S. 102-118, herausgegeben für die Friedrich-Ebert-Stiftung von Ralf Melzer). Bonn: Dietz. $\mathrm{H}$

Groß, E.M., Hövermann, A., \& Messner, S.F. (2018). Marketized Mentalitiy, competitive/egoistic school culture, and delinquent attitudes and behavior: an application of institutional anomie theorie. Criminology, 56(2), 333-369.

Heitmeyer, W. (2002). Gruppenbezogene Menschenfeindlichkeit: Die theoretische Konzeption und erste empirische Ergebnisse. In W. Heitmeyer (Hrsg.), Deutsche Zustände (Folge 1, S. 15-34). Frankfurt a. M.: Suhrkamp.

Heyder, A. (2003). Bessere Bildung, bessere Menschen? Genaueres Hinsehen hilft weiter. In W. Heitmeyer (Hrsg.), Deutsche Zustände (Folge 2, S. 78-99). Frankfurt a. M.: Suhrkamp.

Hooghe, M., \& Wilkenfeld, B. (2008). The stability of political attitudes and behaviors across adolescence and early adulthood: a comparison of survey data on adolescents and young adults in eight countries. Journal of Youth and Adolescence, 37(2), 155-167.

Hopf, W. (1999). Ungleichheit der Bildung und Ethnozentrismus. Zeitschrift für Pädagogik, 45(6), $847-865$.

Hopf, C., \& Hopf, W. (1997). Familie, Persönlichkeit, Politik. Eine Einführung in die politische Sozialisation (Grundlagentexte Soziologie). Weinheim: Juventa.

Hövermann, A., Messner, S.F., \& Zick, A. (2015). Anomie, marketization, and prejudice toward purpotedly unprofitable groups: Elaborating a theoretical approach on anomie-driven prejudices. Acta Sociologica, 58(3), 215-231.

Hughes, C. (2017). Understanding Prejudice and Education. The challenge for future generations. Abingdon, New York: Routledge. 
Infratest dimap (2019a). Landtagswahlen Brandenburg 2019. https://wahl.tagesschau.de/wahlen/2019-0901-LT-DE-BB/index.shtml. Zugegriffen: 14. Jan. 2020.

Infratest dimap (2019b). Landtagswahlen Sachsen 2019. https://wahl.tagesschau.de/wahlen/2019-09-01LT-DE-SN/. Zugegriffen: 14. Jan. 2020.

Infratest dimap (2019c). Landtagswahlen Thüringen 2019. https://wahl.tagesschau.de/wahlen/2019-1027-LT-DE-TH/index.shtml. Zugegriffen: 14. Jan. 2020.

Jäger, S. (1997). Die Anstifter der Brandstifter? Zum Anteil der Medien an der Eskalation rassistisch motivierter Gewalt in der Bundesrepublik Deutschland. In B. Scheffer (Hrsg.), Medien und Fremdenfeindlichkeit. Alltägliche Paradoxien, Dilemmata, Absurditäten und Zynismen (S. 73-98). Opladen: Leske + Budrich.

Kevenhörster, P. (2008). Entscheidungen und Strukturen der Politik (Politikwissenschaft, Bd. 1, 3. Aufl.). Wiesbaden: VS.

Kimmel, M. (2016). Angry White Men. Die USA und ihre zornigen Männer (Schriftenreihe, Bd. 1730). Bonn: Bundeszentrale für politische Bildung.

Kleeberg-Niepage, A. (2012). Zur Entstehung von Rechtsextremismus im Jugendalter - oder: Lässt sich richtiges politisches Denken lernen? Journal für Psychologie, 20(2), 1-30.

Klönne, A. (1994). Jugend und Rechtsextremismus. In W. Kowalsky \& W. Schroeder (Hrsg.), Rechtsextremismus. Einführung und Forschungsbilanz (S. 129-142). Opladen: Westdeutscher Verlag.

KMK (2018). Demokratie als Ziel, Gegenstand und Praxis historisch-politischer Bildung und Erziehung in der Schule (Beschluss der Kultusministerkonferenz vom 06.03.2009 i.d. F. vom 11.10.2018). http:// www.kmk.org/fileadmin/Dateien/pdf/PresseUndAktuelles/2018/Beschluss_Demokratieerziehung. pdf. Zugegriffen: 6. Mai 2020.

Krause, D., \& Faulbaum, F. (2016). Methodische Grundlagen der Erhebung. In A. Zick, B. Küpper \& D. Krause (Hrsg.), Gespaltene Mitte - Feindselige Zustände. Rechtsextreme Einstellungen in Deutschland 2016 (S. 23-31, herausgegeben für die Friedrich-Ebert-Stiftung von Ralf Melzer.). Bonn: Dietz.

Krause, D., \& Zick, A. (2013). Gruppenbezogene Menschenfeindlichkeit: Kurzskalen. In C. J. Kemper, E. Brähler \& M. Zenger (Hrsg.), Psychologische und sozialwissenschaftliche Kurzskalen: Standardisierte Erhebungsinstrumente für Wissenschaft und Praxis (S. 100-136). Berlin: Medizinisch Wissenschaftliche Verlagsgesellschaft.

Krieg, Y., \& Kliem, S. (2019). Rechtsextremismus unter Jugendlichen in Niedersachsen. Ergebnisse repräsentativer Wiederholungsbefragungen aus den Jahren 2013, 2015 und 2017. Monatsschrift für Kriminologie und Strafrechtsreform, 102(2), 135-153.

Kuhn, H.-P., \& Krappmann, L. (Hrsg.). (2000). Sozialisation zur Mitbürgerlichkeit. Wiesbaden: Springer.

Küpper, B., Berghan, W., \& Rees, J.H. (2019b). Aufputschen von Rechts: Rechtspopulismus und seine Normalisierung in der Mitte. In A. Zick, B. Küpper \& W. Berghan (Hrsg.), Verlorene Mitte - Feindselige Zustände. Rechtsextreme Einstellungen in Deutschland 2018/19 (S. 173-202, herausgegeben für die Friedrich-Ebert-Stiftung von Franziska Schröter.). Bonn: Dietz.

Küpper, B., Krause, D., \& Zick, A. (2019a). Rechtsextreme Einstellungen in Deutschland 2002-2018/19. In A. Zick, B. Küpper \& W. Berghan (Hrsg.), Verlorene Mitte - Feindselige Zustände. Rechtsextreme Einstellungen in Deutschland 2018/19 (S. 117-146, herausgegeben für die Friedrich-Ebert-Stiftung von Franziska Schröter.). Bonn: Dietz.

Küpper, B., Schröter, F., \& Zick, A. (2019c). Alles nur ein Problem der Ostdeutschen oder Einheit in Wut und Hass? Rechtsextreme und menschenfeindliche Einstellungen in Ost- und Westdeutschland. In A. Zick, B. Küpper \& W. Berghan (Hrsg.), Verlorene Mitte - Feindselige Zustände. Rechtsextreme Einstellungen in Deutschland 2018/19 (S. 243-282, herausgegeben für die Friedrich-Ebert-Stiftung von Franziska Schröter). Bonn: Dietz.

Landeskriminalamt Sachsen-Anhalt (2019). Jugendkriminalität und Jugendgefährdung im Land SachsenAnhalt Jahresbericht 2018. Magdeburg: Landeskriminalamt Sachsen-Anhalt.

Langebach, M. (2016). Rechtsextremismus und Jugend. In F. Virchow, M. Langebach \& A. Häusler (Hrsg.), Handbuch Rechtsextremismus (Edition Rechtsextremismus, S. 375-439). Wiesbaden: Springer VS.

Lozano, L. M., García-Cueto, E., \& Muñiz, J. (2008). Effect of the number of response categories on the reliability and validity of rating scales. Methodology, 4(2), 73-79.

Lubke, G.H., \& Muthén, B.O. (2009). Applying multigroup confirmatory factor models for continuous outcomes to Likert scale data complicates meaningful group comparisons. Structural Equation Modeling, 11(4), 514-534. 
Lynen von Berg, H. (2000). Politische Mitte und Rechtsextremismus. Diskurse zu fremdenfeindlicher Gewalt im 12. Deutschen Bundestag (1990-1994) (Forschung Politikwissenschaft, Bd. 72). Opladen: Leske + Budrich.

Ministerium des Innern des Landes Nordrhein-Westfalen (2019). Verfassungsschutzbericht des Landes Nordrhein-Westfalen über das Jahr 2018. Düsseldorf: Ministerium des Innern des Landes NordrheinWestfalen.

Mudde, C. (2014). Youth and the extreme right: explanations, issues, and solutions. In C. Mudde (Hrsg.), Youth and the extreme right (S. 1-18). New York: Idebate Press.

Mudde, C., \& Kaltwasser, C. R. (2017). Populism. A very short introduction. New York: Oxford University Press.

Ostapczuk, M., Musch, J., \& Moshagen, M. (2008). A randomized-response investigation of the education effect in attitudes towards foreigners. European Journal of Social Psychology, 39(6), 920-931.

Preiser, S. (2008). Jugend und Politik: Anpassung - Partizipation - Extremismus. In R. Oerter \& L. Montada (Hrsg.), Entwicklungspsychologie (Bd. 6, S. 874-884). Weinheim: Beltz.

Raabe, T., \& Beelmann, A. (2011). Development of ethnic, racial, and national prejudice in childhood and adolescence: a multinational meta-analysis of age differences. Child Development, 82(6), 1715-1737.

Reinders, H. (2001). Politische Sozialisation Jugendlicher in der Nachwendezeit. Forschungsstand, theoretische Perspektiven und empirische Evidenzen (Forschung Erziehungswissenschaft, Bd. 132). Opladen: Leske + Budrich.

Rieker, P. (2006). Rechtsextremismus - Ein Jugendproblem? Altersspezifische Befunde und forschungsstrategische Herausforderungen. Diskurs Kindheits- und Jugendforschung, 1(2), 245-260.

Rippl, S. (2008). Politische Sozialisation. In K. Hurrelmann, M. Grundmann \& S. Walper (Hrsg.), Handbuch Sozialisationsforschung (Bd. 7). Weinheim: Beltz.

Rippl, S., Seipel, C., \& Kindervater, A. (2015). Politische Sozialisation. In S. Zmerli \& O. Feldman (Hrsg.), Politische Psychologie. Handbuch für Studium und Wissenschaft (Politische Psychologie: Themen, Herausforderungen, Perspektiven, Bd. 1, S. 69-84). Baden-Baden: Nomos.

Schenke, J., Schmitz, C., Marg, S., \& Trittel, K. (2018). PEGIDA-Effekt? Jugend zwischen Polarisierung und politischer Unberührtheit. Bielefeld: transcript.

Schneekloth, U., \& Albert, M. (2019). Jugend und Politik: Demokratieverständnis und politisches Interesse im Spannungsfeld von Vielfalt, Toleranz und Populismus. In Shell Deutschland Holding (Hrsg.), Jugend 2019. Eine Generation meldet sich zu Wort (18. Shell Jugendstudie, S. 47-101). Weinheim: Beltz.

Schütz, H., \& Six, B. (1996). How strong is the relationship between prejudice and discrimination? A meta-analytic answer. International Journal of Intercultural Relations, 20(3-4), 441-462.

Stöss, R. (1989). Die extreme Rechte in der Bundesrepublik. Entwicklung - Ursachen - Gegenmaßnahmen. Opladen: Westdeutscher Verlag.

Stöss, R. (2007). Rechtsextremismus im Wandel (2., aktualisierte Aufl.). Berlin: Friedrich-Ebert-Stiftung. http://library.fes.de/pdf-files/do/05227.pdf. Zugegriffen: 17. Okt. 2019.

Stöss, R., \& Niedermayer, O. (2008). Rechtsextreme Einstellungen in Berlin und Brandenburg 2000-2008 sowie in Gesamtdeutschland 2005 und 2008. https://www.polsoz.fu-berlin.de/polwiss/forschung/ systeme/empsoz/team/ehemalige/Publikationen/media/rex_00_08.pdf. Zugegriffen: 17. Okt. 2019.

Talaska, C. A., Fiske, S. T., \& Chaiken, S. (2008). Legitimating racial discrimination: emotions, not beliefs, best predict discrimination in a meta-analysis. Social Justice Research, 21(3), 263-296.

Wahl, K., Tramitz, C., \& Blumtritt, J. (2001). Fremdenfeindlichkeit. Auf den Spuren extremer Emotionen. Opladen: Leske + Budrich.

Würstl, H. (2016). Uwe Böhnhardt. Rekonstruktion einer kriminellen Karriere. In W. Frindte, D. Geschke, N. Haußecker \& F. Schmidtke (Hrsg.), Rechtsextremismus und „Nationalsozialistischer Untergrund“. Interdisziplinäre Debatten, Befunde und Bilanzen (Edition Rechtsextremismus, S. 213-224). Wiesbaden: Springer VS.

Zick, A., \& Klein, A. (2014). Fragile Mitte - Feindselige Zustände. Rechtsextreme Einstellungen in Deutschland 2014 (herausgegeben für die Friedrich-Ebert-Stiftung von Ralf Melzer). Bonn: Dietz.

Zick, A., \& Küpper, B. (2006). Politische Mitte. Normal feindselig. In W. Heitmeyer (Hrsg.), Deutsche Zustände (Folge 4, S. 115-134). Frankfurt a. M.: Suhrkamp.

Zick, A., \& Küpper, B. (2018). Menschenfeindliche Vorurteile im Kontext von Radikalisierungsdynamiken und rechtsextremen Handlungen. Monatszeitschrift für Kriminologie und Strafrechtsreform, 101(2), $140-171$.

Zick, A., Berghan, W., \& Mokros, N. (2019b). Gruppenbezogene Menschenfeindlichkeit in Deutschland 2002-2018/19 (mit einem Exkurs zum neuen Antisemitismus von Beate Küpper und Andreas Zick). In A. Zick, B. Küpper \& W. Berghan (Hrsg.), Verlorene Mitte - Feindselige Zustände. Rechtsextreme 
Einstellungen in Deutschland 2018/19 (S. 53-116, herausgegeben für die Friedrich-Ebert-Stiftung von Franziska Schröter). Bonn: Dietz.

Zick, A., Küpper, B., \& Berghan, W. (2019a). Verlorene Mitte - Feindselige Zustände. Rechtsextreme Einstellungen in Deutschland 2018/19 (herausgegeben für die Friedrich-Ebert-Stiftung von Franziska Schröter). Bonn: Dietz.

Zick, A., Küpper, B., \& Krause, D. (2016). Gespaltene Mitte - Feindselige Zustände. Rechtsextreme Einstellungen in Deutschland 2016 (herausgegeben für die Friedrich-Ebert-Stiftung von Ralf Melzer). Bonn: Dietz.

Zick, A., Rees, J., Papandick, M., \& Wäschle, F. (2018). MEMO. Multidimensionaler Erinnerungsmonitor (Studie 1). Bielefeld: Institut für interdisziplinäre Konflikt- und Gewaltforschung.

Zick, A., Wolf, C., Küpper, B., Davidov, E., Schmidt, P., \& Heitmeyer, W. (2008). The syndrome of groupfocused enmity: the interrelation of prejudices tested with multiple cross-sectional and panel data. Journal of Social Issues, 64(2), 363-383. 